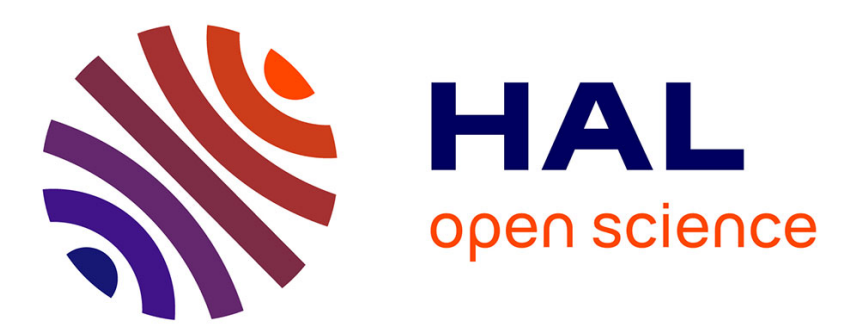

\title{
Reality-Based Real-Time Cell Indentation Simulator
}

Hamid Ladjal, Jean-Luc Hanus, Anand Pillarisetti, Carol Keefer, Antoine

Ferreira, Jaydev P.Desai

\section{To cite this version:}

Hamid Ladjal, Jean-Luc Hanus, Anand Pillarisetti, Carol Keefer, Antoine Ferreira, et al.. RealityBased Real-Time Cell Indentation Simulator. IEEE/ASME Transactions on Mechatronics, 2012, 17 (2), pp.239 - 250. 10.1109/TMECH.2010.2091010 . hal-00647908

\section{HAL Id: hal-00647908 https://hal.science/hal-00647908}

Submitted on 3 Dec 2011

HAL is a multi-disciplinary open access archive for the deposit and dissemination of scientific research documents, whether they are published or not. The documents may come from teaching and research institutions in France or abroad, or from public or private research centers.
L'archive ouverte pluridisciplinaire HAL, est destinée au dépôt et à la diffusion de documents scientifiques de niveau recherche, publiés ou non, émanant des établissements d'enseignement et de recherche français ou étrangers, des laboratoires publics ou privés. 
- Reality-Based Real-Time Cell Indentation Simulator

\author{
Hamid Ladjal, Jean-Luc Hanus, Anand Pillarisetti, Carol Keefer, Antoine Ferreira, Member, IEEE, \\ and Jaydev P. Desai, Senior Member, IEEE
}

Abstract-Training simulators that provide realistic visual and haptic feedback during cell indentation tasks are currently investigated. Complex cell geometry inherent to biological cells and intricate mechanical properties drive the need for precise mechanical and numerical modeling to assure accurate cell deformation and force calculations. Advances in alternative finite-element formulation, such as the mass-tensor approach, have reached a state, where they are applicable to model soft-cell deformation in real time. The geometrical characteristics and the mechanical properties of different cells are determined with atomic force microscopy (AFM) indentation. A real-time, haptics-enabled simulator for cell centered indentation has been developed, which utilizes the AFM data (mechanical and geometrical properties of embryonic stem cells) to accurately replicate the indentation task and predict the cell deformation during indentation in real time. This tool can be used as a mechanical marker to characterize the biological state of the cell. The operator is able to feel the change in the stiffness during cell deformation between fixed and live cells in real time. A comparative study with finite-element simulations using a commercial software and the experimental data demonstrate the effectiveness of the proposed physically based model.

Index Terms-Atomic force microscope (AFM), finite-element modeling (FEM), haptics, modeling, real-time interaction, stem cell.

Manuscript received March 31, 2010; revised July 7, 2010 and October 5, 2010; accepted October 8, 2010. Recommended by Technical Editor Y. Sun. A portion of this paper was presented at the IEEE/RSJ International Conference on Intelligent Robots and Systems, Taipei, Taiwan, October 18-22, 2010. This work was supported by the National Science Foundation under Grant CMMI 0826158, in part by STMD-Maryland Technology Development Corporation under Grant 08071517, and in part by the Centre de Recherche en Biologie de Baugy, France.

H. Ladjal, J.-L. Hanus, and A. Ferreira are with the Institut PRISME, Ecole Nationale Supérieure d'Ingénieurs de Bourges, 18000 Bourges, France (e-mail: hamid.ladjal@ensi-bourges.fr; jean-luc.hanus@ensi-bourges.fr; antoine.ferreira@ensi-bourges.fr).

A. Pillarisetti is with the Department of Mechanical Engineering and Applied Mechanics, University of Pennsylvania, Philadelphia, PA 19104 USA (e-mail :anandpi@seas.upenn.edu.)

C. Keefer is with the Department of Animal and Avian Sciences, University of Maryland, College Park, MD 20742 USA (e-mail: ckeefer@umd.edu).

J. P. Desai is with the Robotics, Automation, and Medical Systems (RAMS) Laboratory, Maryland Robotics Center, Institute for Systems Research, University of Maryland, College Park, MD 20742 USA (e-mail: jaydev@ umd.edu).

Color versions of one or more of the figures in this paper are available online at http://ieeexplore.ieee.org.

Digital Object Identifier 10.1109/TMECH.2010.2091010

\section{The Promise of Stem Cell Research}

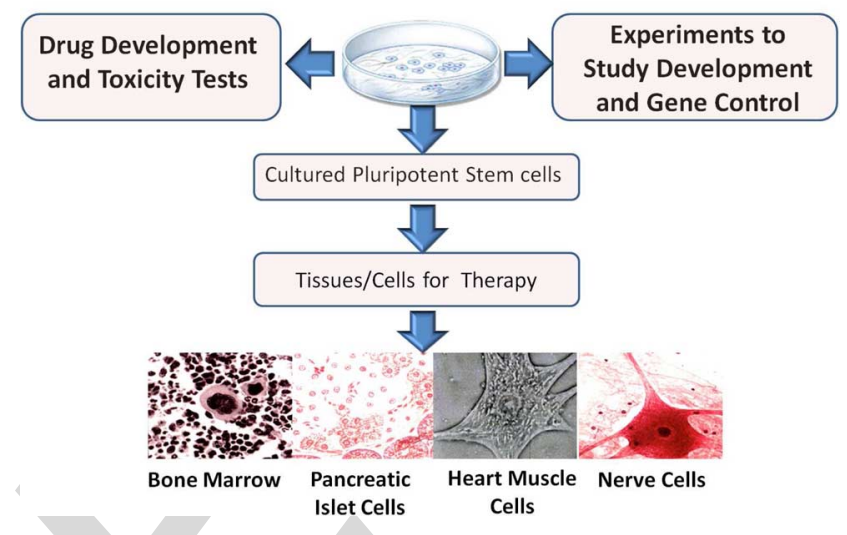

Fig. 1. Stem cells are valuable resources for toxicology, developmental biology, and regenerative medicine research.

important role in cell development and differentiation [1]-[3]. In contrast to adult stem cells, embryonic stem cells differentiate into every cell type in the body, and thus, have a huge therapeutic potential. Stem-cell-based systems offer a very promising and innovative solution for obtaining large number of cells for early efficacy and toxicity screening. Stem cell technology provides a new tool for drug development and a better insight to understand mammalian gene function (see Fig. 1). The mechanical properties of biological cells have been studied with different techniques [4], the most popular being optical tweezers [5], magnetic beads [6], and micropipette aspiration [7]. Different microrobotic systems with various end effectors have been proposed [33], [34]. However, these methods cannot compete with the precision that can be obtained with the atomic force microscopy (AFM) method [8], [32]. AFMs have been widely used in the study of micro- and nanostructures including living cells. Modern AFM techniques allow solving several problems of cell biomechanics by simultaneous evaluation of the local mechanical properties and the topography of living cells, at a high spatial resolution and force sensitivity [9]. In these experiments, an AFM cantilever serves as a microindenter to probe the cell, and further analysis of force-indentation data yields the local Young's modulus. In addition, the AFM indentation technique can be used to characterize the viscoelastic behavior of the cell cytoskeleton [10], including viscosity [11], loss and storage moduli [12], and stress relaxation times. Mechanical phenotyping prove actually to be a valuable tool in the development of improved methods of targeted cellular differentiation of embryonic, induced pluripotent (iPS), and adult stem cells for therapeutic and diagnostic purposes [30], [31]. In this study, we propose to characterize the mechanical behavior of an individual mouse embryonic stem cells (mESC) in undifferentiated state 
using AFM indentation [13]. As we hypothesize that the cell stiffness of mESC may reflect the nuclear physical changes of the cell, it should be possible to detect modifications in stiffness by probing the cell directly. However, different limitations are inherent in AFM-based indentation techniques. First, deformations induced by the AFM probe and cell positions cannot be seen by the user. Second, the force sensing of the AFM cannot resolve the force interaction between the AFM tip and the cell's surface independently in all three dimensions to avoid cytoskeleton-disrupted experiments. Therefore, the user performs extremely challenging manipulations requiring advanced manual skills. Modeling the physics of the interaction between tip and cell is necessary to realize complicated indentation tasks like cancer cell probing [14].

We developed finite-element models (FEMs) that include the topological information of adherent stem cells (shape and dimensions), AFM tips (conical and spherical), and biological structure (cell models with cytoplasm layers, cytoskeletons, and nucleus) [15]. To validate the viscoelastic and nonlinear dynamics of the cell in real time using finite-element procedures, we report herein the mechanical characterization of single mESC indentation-relaxation for different colonies of fixed and live undifferentiated mESCs (termed, undiff $m E S C$ ). The proposed indentation tests use microsphere-modified AFM probes in order to estimate the global elastic modulus of the cell to reflect the true global response of a mESC [14], [15]. This paper is a subsequent contribution to [27], where various analytical models are tested and compared to estimate the mechanical properties of the biological cells. Based on the proposed physical model, we have developed a computer-based training system using force feedback to simulate cell indentation procedures in virtual environments (VEs) for biologist training. The paper consists of five sections. In Section II, we present the biomechanical finite-element approach dedicated to real-time indentation. In Section III, we present the methodology of the VE system for cell indentation. In Section IV, we present the experimental setup using the force microscopy. In Section V, we make a comparative study between the physics-based model and experimental data. In Section VI, we present our real-time cell indentation simulator using a spherical tip. Finally, we give some concluding remarks and the directions for future work.

\section{Physics-Based Deformable Models for Cell NANOINDENTATION SIMULATION}

In this section, we present further developments to the linear elastic mass-tensor model introduced by [16], [17] and extended by Schwartz et al. [18]. In our paper, the cell is modeled by a volume object discretized into a conformal tetrahedral mesh as defined by finite-element theory.

Using classical notations, inside each tetrahedron $T^{k}$, the displacement field, defined by a linear interpolation $\left[\mathbf{N}^{\mathrm{k}}\right]$ of the nodal displacement vector $\left\{\mathbf{u}^{\mathrm{k}}\right\}$ of the four vertices of tetrahedron, is written as follows:

$$
\left\{\mathbf{U}(\mathbf{x})^{\mathbf{k}}\right\}=\left[\mathbf{N}^{\mathbf{k}}(\mathbf{x})\right] \quad\left\{\mathbf{u}^{\mathbf{k}}\right\}
$$

For a linear elastic material, the relation between the Cauchy 118 stress tensor and the linearized strain tensor is written with 119 Lame's coefficient in condensed vector notation as follows:

$$
\{\boldsymbol{\sigma}\}=\lambda\left(\{\varepsilon\}_{1}+\{\varepsilon\}_{2}+\{\varepsilon\}_{3}\right)[\mathbf{I}]+2 \mu\{\varepsilon\}
$$

where $[\mathbf{I}]$ is the identity matrix.

The principle of virtual work applied to a single tetrahadron $T^{k}$ leads to the elementary stiffness matrix $\left[\mathbf{K}^{\mathbf{k}}\right]$ such that the elementary nodal force vector acting on a tetrahedron is as follows:

$$
\left\{\mathbf{f}^{\mathrm{k}}\right\}=\left[\mathbf{K}^{\mathrm{k}}\right]\left\{\mathbf{u}^{\mathrm{k}}\right\} .
$$

This stiffness matrix is composed of a plurality of elementary submatrices, each connecting the elementary force acting on the node $i$ to the displacement of the node $j$

$$
\begin{aligned}
{\left[\mathbf{K}_{\mathbf{i j}}^{\mathbf{k}}\right]=\frac{1}{36 V^{k}} } & \left(\lambda\left\{\mathbf{m}_{\mathbf{i}}\right\}\left\{\mathbf{m}_{\mathbf{j}}\right\}^{T}\right. \\
& \left.+\mu\left\{\mathbf{m}_{\mathbf{j}}\right\}\left\{\mathbf{m}_{\mathbf{i}}\right\}^{T}+\mu\left\{\mathbf{m}_{\mathbf{i}}\right\}^{T}\left\{\mathbf{m}_{\mathbf{j}}\right\}[\mathbf{I}]\right)
\end{aligned}
$$

where $\{\mathbf{m}\}$ are unit outward-pointing normals to triangular faces and $V^{k}$ is the volume of the tetrahedron $T^{k}$.

Taking into account the contribution of all adjacent tetrahedra, the global internal force acting on a node $l$ can be expressed as follows:

$$
\left\{\mathbf{F}_{\mathrm{int}}^{\mathbf{l}}\right\}=\sum_{k \in \mathcal{V}_{l}}\left(\sum_{j=1}^{4}\left[\mathbf{K}_{\mathbf{i j}}^{\mathbf{k}}\right]\left\{\mathbf{u}_{\mathbf{j}}\right\}\right)
$$

where $\mathcal{V}_{l}$ is the neighborhood of vertex $l$ (i.e., the tetrahedra 134 containing node $l$ ).

The tensors $\left[\mathbf{K}_{\mathrm{ij}}^{1}\right]$ depending on the remaining geometry and Lame's coefficients are constant. They can be precomputed in an offline phase. This is the essential advantage of the mass-tensor approach, which makes it useful for real-time application.

\section{A. Cell Modeling Using FEM}

In our paper, the mESC is meshed with 3-D first-order tetrahedral elements, as shown in Fig. 2. The geometrical characteristics of the cells were obtained from experimental data using the AFM system. This includes shape, diameter, and height of the cells. The mesh that has been used for the following simulations is composed of 835 vertices and 2456 tetrahedra, where all vertices are free to move except the ones in contact with the petri dish, which are fixed.

\section{B. Dynamic Model}

The equation of motion of a vertex $l$ of the cell mesh can be written as follows:

$$
M^{l}\left\{\ddot{\mathbf{u}}_{l}\right\}+\gamma^{l}\left\{\dot{\mathbf{u}}_{l}\right\}+\sum_{k \in \mathcal{V}_{l}}\left(\sum_{j=1}^{4}\left[\mathbf{K}_{\mathbf{i j}}^{\mathbf{k}}\right]\left\{\mathbf{u}_{\mathbf{j}}\right\}\right)=\left\{\mathbf{F}_{\text {ext }}^{\mathbf{l}}\right\}
$$

where $M^{l}$ and $\gamma^{l}$ are, respectively, the mass and damping coef- 152 ficients of each vertex. 


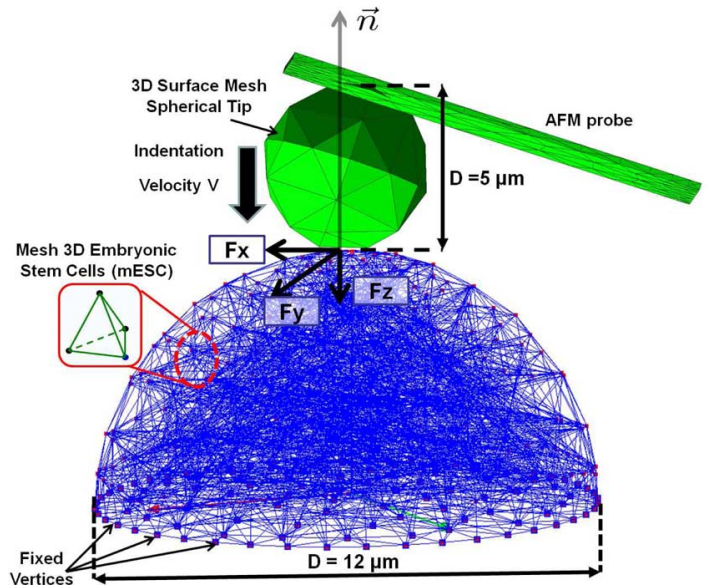

Fig. 2. Representation of the 3-D mESC mesh using P1 tetrahedral finite elements.

To solve the dynamic system, we tested different integration schemes (implicit and explicit) taking into account the tradeoff between real-time simulation and haptic stability requirements. We choose the explicit centered finite-difference scheme defined by

$$
\left\{\begin{array}{l}
u(t+h)=u(t)+\dot{u}(t) h+\frac{1}{2} \ddot{u}(t) h^{2}+O\left(h^{3}\right) \\
\dot{u}(t+h)=\dot{u}(t)+\frac{1}{2} \ddot{u}(t) h+\frac{1}{2} \ddot{u}(t+h) h+O\left(h^{3}\right)
\end{array}\right.
$$

where $h$ is the temporal integration step size chosen to satisfy the Courant-Friedrich-Lewy condition.

The discrete equation of motion of a vertex $l$ reads

$$
\begin{aligned}
\left(M^{l}+\frac{h}{2} \gamma^{l}\right)\left\{\ddot{\mathbf{u}}_{l}\right\}_{(t+h)} \\
=\left\{\mathbf{F}_{\text {ext }}^{\mathbf{l}}\right\}_{(t+h)}-\gamma^{l}\left(\left\{\dot{\mathbf{u}}_{l}\right\}_{(t)}+\frac{h}{2}\left\{\ddot{\mathbf{u}}_{l}\right\}_{(t)}\right) \\
\quad-\sum_{k \in \mathcal{V}_{l}}\left(\sum_{j=1}^{4}\left[\mathbf{K}_{\mathbf{i j}}^{\mathbf{k}}\right]\left(\left\{\mathbf{u}_{\mathbf{j}}\right\}_{(t)}+h\left\{\dot{\mathbf{u}}_{j}\right\}_{(t)}+\frac{h^{2}}{2}\left\{\ddot{\mathbf{u}}_{j}\right\}_{(t)}\right)\right)
\end{aligned}
$$

from which we can compute the new vertices for accelerations, velocities, and positions.

\section{VE SYSTEM FOR CELL INDENTATION}

Fig. 3 shows the architecture of the real-time virtual-realitybased cell indentation simulator. This includes the computer generated models of stem cells, the AFM nanoindentor, the collision detection algorithm, physics-based models of deformable $\mathrm{mESC}$, and the haptic interaction controller. The operator will be able to interact with the 3-D model of the cell using his sense of vision as well as actively manipulate using his sense of touch. Usually, real-time graphics translates to an update rate of $25 \mathrm{~Hz}$, stable haptic interaction in VE's requires much higher update rate of around $1 \mathrm{kHz}$. As soft biological tissues exhibit complex viscoelastic and nonlinear properties, the realtime interaction imposes severe restrictions on the FEM model.

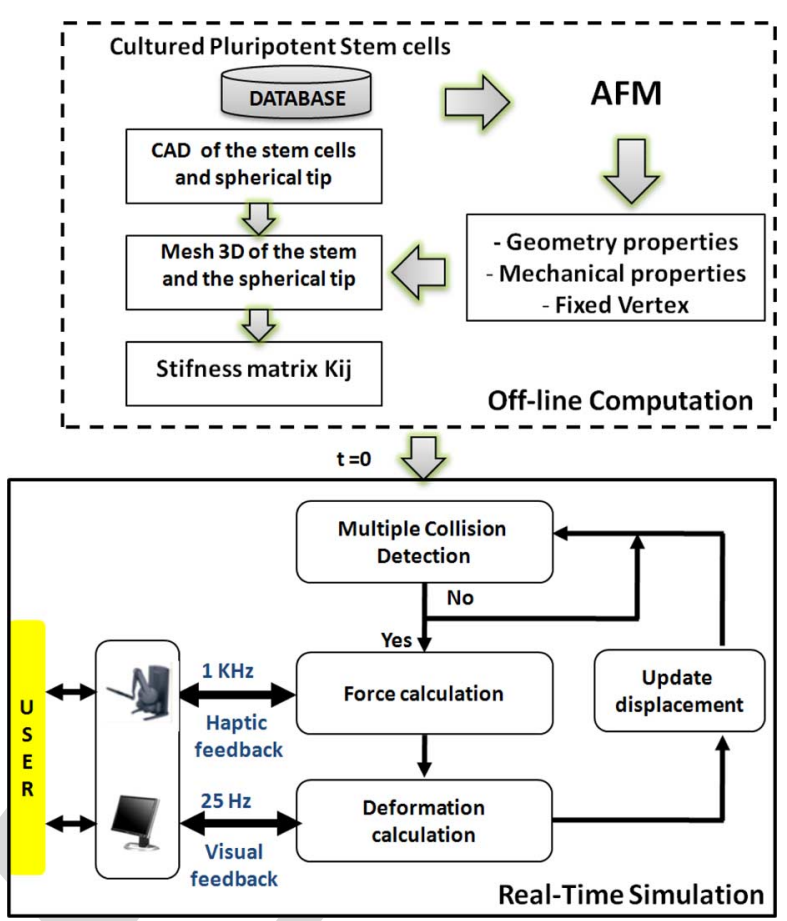

Fig. 3. Computational architecture for simulating force-reflecting deformable cell indentation in a VE. The figure shows the two simulation phases used for the real-time indentation of the cell: 1) offline precalculations of stiffness matrices and 2) simulation of visual and haptic interaction.

A significant difficulty of using the finite-element technique for 177 real-time simulation is that it is computationally costly. These 178 mesh-based schemes also require an expensive numerical in- 179 tegration operation for the computation of the system stiffness 180 matrices. For this reason, we adopted a computational architec- 181 ture with two simulation stages: 1) an offline computation of 182 stiffness matrices for each triangular element and 2) visual and 183 haptic interaction for real-time indentation simulation.

\section{A. Offline Computation}

The proposed computational methodology contains an offline precalculation step. The most costly and time-consuming operations are realized during this step. The database contains various developed models of the different stem cells geometry (bone marrow, pancreatic, heart muscle, nerve cells, etc.). Using AFM image analysis (ImagePro software from AFM Asylum), we derived different geometric stem cell models based on a commercial computer-aided design (CAD) package (MARC-ADAMS). The meshing of the 3-D internal structure of the mESC is then carried out through a dedicated 3-D meshing software (GID software) modeled in exact dimensions. Although they were displayed as 3-D texture-mapped objects to the user, they were modeled as connected line segments to reduce the number of collision computations during real-time interactions.

The adherent cell was approximated as an assembly of discrete tetrahedral elements interconnected to each other through a fixed number of nodes (see Fig. 2). The displacements of these nodal points for applied external indentation forces were the basic unknowns of our FEM analysis. The coordinates 


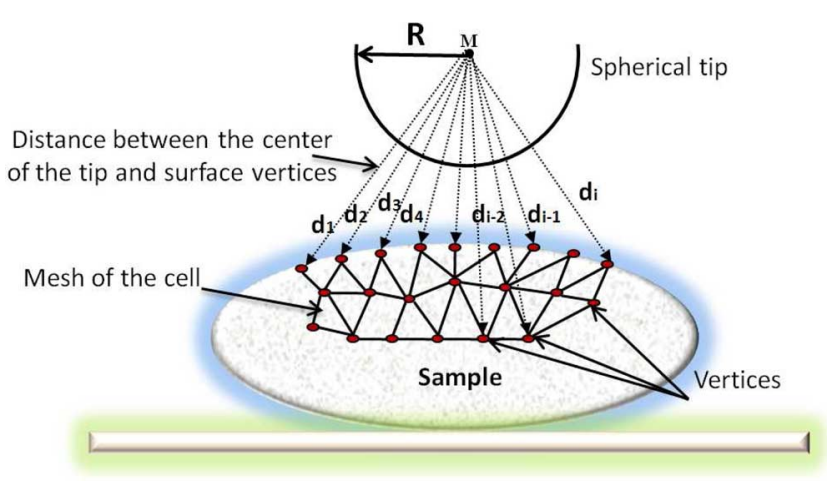

Fig. 4. Scheme illustrating the multiple collision detection algorithm using a simple sphere-points intersection problem: the distance between the center of the spherical tip and the nodes of the cell surface is calculated in real time.

of vertices, the tetrahedron indexing, and the connectivity of vertices were derived from the geometric model. Then, the mechanical (Young's modulus and stiffness) and geometrical (height and shape) properties of the cell structure are determined through AFM indentation experiments. Finally, these properties are passed to the FEM algorithm for calculating the physically based behavior of biological soft tissues. The offline calculation of all tensors $K_{i j}$ allows precalculation stiffness values for preselected cells such as, for example, live and fixed cells.

\section{B. Real-Time Indentation}

Since haptics and graphics have different update frequencies, we implemented separate threads to update the loops. During the indentation task, the contact between the tip and the cell must occur at a special set of points (nodal points).

1) Collision Detection: An important part of biological cantilever tip-soft cell modeling is the fast collision detection algorithm. The collision detection is the first step to carry out realistic interactions. However, locating the contact primitive (e.g., facets) between two objects may be computationally expensive, especially if the objects are composed of a large number of polygons. In general, to determine a contact between two virtual objects, we compute the distance between them. If this distance is negative, then the objects are in contact. We may continue by determining the set of geometric objects or entities that collide. The cantilever tip in VE can be modeled as a complex 3-D object, composed of numerous surfaces, edges, and vertices, but this constitutes a challenging task. For the purpose of detecting collisions, we used a point-based representation of the cantilever tip as a simple spherical object and employed a simple sphere-points intersection algorithm with local search technique. It calculates the distance between the center of the spherical tip and the nodes of the cell surface (see Fig. 4).

2) Penalty-Based Models: In an interaction model, a collision between two objects may generate local and global deformations of the colliding objects, new internal forces, changes in the velocities of the involved objects, and topology modifications, such as punction or cutting. Physical simulations suppose that these events are related to the mechanical properties of the objects. In our simulations, we used the penalty-based models.

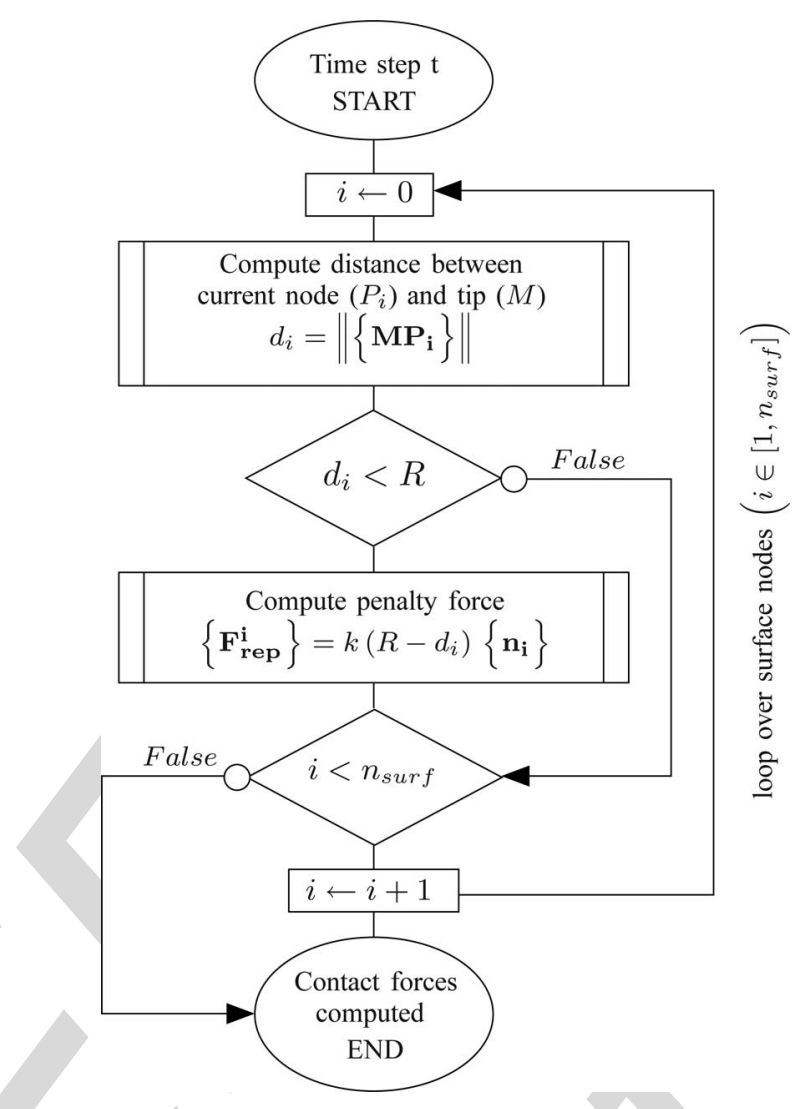

Fig. 5. Main flowchart for multi collision detection: $M$ represents the position of the center of the spherical tip and $R$ its radius, $i$ represents the current external cell surface node, $d_{i},\left\{F_{\text {rep }}^{i}\right\}$ and $\left\{n_{i}\right\}$ represent, respectively, the computed distance between the center of the tip and the current node, the repulsive force, and the outward-pointing contact normal at node $i$.

These models consider the reactive force that first reduces the 244 relative speed of the two objects, and finally, repulses them from 245 each other, as proportional to the deformation. Thus, the reac- 246 tive force is a function of the local interpenetration depth $\delta$. The 247 normal repulsive force $F_{\text {rep }}$ can be written as follows:

$$
\left\{F_{\text {rep }}\right\}=\lambda \delta\{n\}
$$

where $\lambda$ is the penalty of the interpenetration and $\{n\}$ is the 249 outward-pointing normal.

These contact forces are evaluated at each time step $t$ (see Fig. 5). They represent the new updated external forces acting upon our stem cell.

3) Virtual Coupling for Stability of the Haptic Rendering: The internal operating loop of the haptic interface requires an update frequency around $1 \mathrm{kHz}$. However, the FEM update frequency is in the range of $25-30 \mathrm{~Hz}$. This frequency difference threatens the coherence between both systems leading to instabilities of the user haptic rendering. We adopted the solution to use a virtual coupling model defined in [24]. This approach introduces a virtual passive link between the 261 simulation model and the haptic interface in order to ensure 262 the stability and the performance of the system [see Fig. 6(a)]. 263 When we combine the impedance display implementation with 264 an appropriate virtual coupling network, we get the admittance 265 


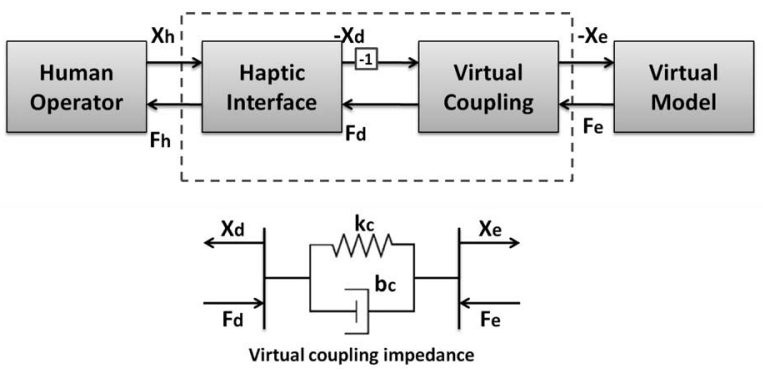

(a)

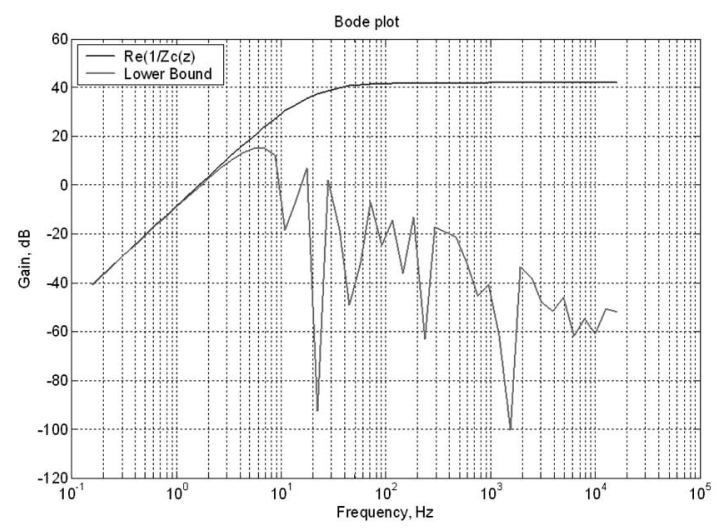

(b)

Fig. 6. Stability conditions using the virtual coupling model. (a) Model of virtual coupling. (b) Impedance display virtual coupling.

matrix for the combined interface. The linear two-port is said to be absolutely stable if there exists no set of passive terminating one-port impedances for which the system is unstable. Llewellyn's stability criteria [25] provides both necessary and sufficient conditions for absolute stability of linear twoports. We get the conditions for absolute stability of the haptic interface

$$
\operatorname{Re}\left(Z_{\mathrm{di}}(z)\right) \geq 0, \frac{1}{Z_{\mathrm{cvi}}(z)} \geq 0
$$

$$
\cos (\angle \mathrm{ZOH}(z))+\frac{2 \operatorname{Re}\left(Z_{\mathrm{di}}(z)\right) \operatorname{Re}\left(1 / Z_{\mathrm{cvi}}(z)\right)}{|\mathrm{ZOH}(z)|} \geq 1
$$

where $Z_{\mathrm{cvi}}(z)$ is the virtual coupling impedance $\left(k_{c}, b_{c}\right)$, $\mathrm{ZOH}(z)$ is a zero-order holder, and $Z_{\mathrm{di}}(z)$ is the PHANToM impedance. The inequality (11) can be rewritten to get an explicit expression of absolute stability of the haptic interface

$$
\operatorname{Re}\left(\frac{1}{Z_{\mathrm{cvi}}(z)}\right) \geq \frac{1-\cos (\angle \mathrm{ZOH}(z))}{2 \operatorname{Re}\left(Z_{\mathrm{di}}(z)\right)}|\mathrm{ZOH}(z)| .
$$

For the virtual coupling, the impedance display induces a limit on the maximum impedance which can be rendered. We use (12) to find the virtual coupling, which makes the haptic interface absolutely stable. Using the design bound of the best performing, absolutely stabilizing virtual coupling. These parameters are found as $b_{c}=0.008 \mathrm{~N} /\left(\mathrm{mm} / \mathrm{s}\right.$ ) and $k_{c}=2.3 \mathrm{~N} / \mathrm{mm}$ (plotted on Fig. 6(a) as a thin red line). The left side of (12) with the resulting values is plotted on Fig. 6(b) as a bold blue line.

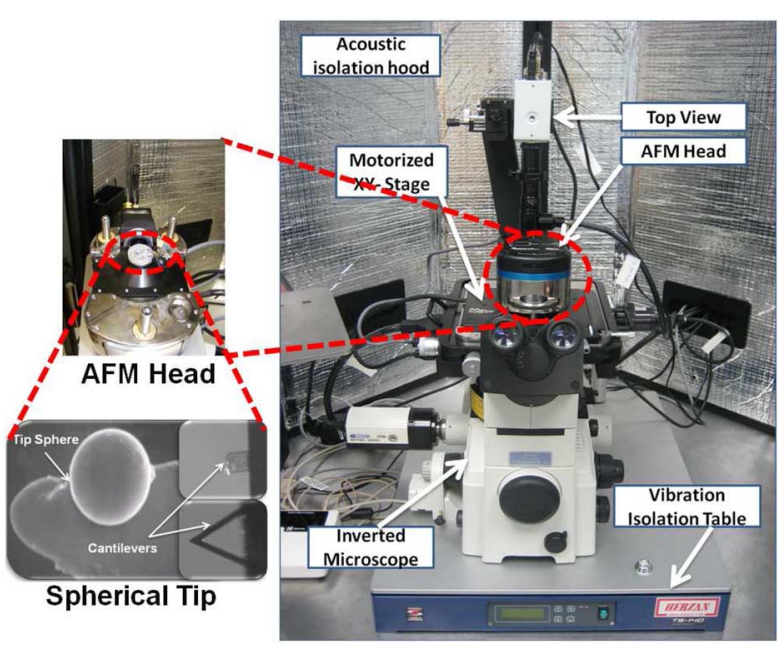

Fig. 7. Experimental setup based on AFM system for mESC indentation studies. The nanoindentor is constituted by a spherical probe ( $5 \mu \mathrm{m}$ diameter) attached to a silicon nitride cantilever from Novascan Technologies.

\section{EXPERIMENTAL SETUP}

\section{A. Description}

The experimental tests of the cell indentations were performed using an AFM (Model: MFP-3D-BIO, Asylum Research, Santa Barbara, CA). The AFM is integrated with a top view module and mounted on an active vibration isolation table manufactured by Herzan (Laguna Hills, CA), as shown in Fig. 7. The top view module enables viewing of cells and easy alignment of the laser beam on the AFM cantilever. The XY stage (manual) allows the user to position the cell beneath the cantilever tip of the AFM. The entire AFM setup is enclosed in an acoustic isolation chamber to prevent acoustic noise from interfering with the AFM measurements. The $x$ - and $y$-axis ranges of the scan head are $90 \mu \mathrm{m}$. The $z$-axis scan range is $40 \mu \mathrm{m}$. The AFM system is used to obtain force and cell deformation data from biological samples.

The cantilever is moved by the piezoelectric scanner in $z$ direction toward the cell. The deflection of the cantilever is detected by a photodiode when the tip comes in contact with the cell. When the tip of the cantilever is in contact with the cell, the initial cantilever deflection $\left(d_{0}\right)$, and initial cantilever movement in $(z)$ direction $\left(z_{0}\right)$ are stored. As the cantilever moves further in $z$-direction and deforms the cell, the final cantilever deflection $\left(d_{1}\right)$ and the cantilever movement $\left(z_{1}\right)$ are obtained [21].

The stiffness depends not only on the Young's modulus but also on the geometry of the tip-surface contact. Therefore, the geometry and spring constant of the cantilever are calibrated in the same way for live and fixed cells. In the following experiments, we employed two types of cantilevers attached with a spherical probe ( $5 \mu \mathrm{m}$ in diameter).

1) Silicon nitride cantilever with a spring constant of 0.06 $\mathrm{N} / \mathrm{m}$ (Novascan Technologies, Inc., Ames, IA) for live cells.

2) Silicon cantilever with a spring constant of $1.75 \mathrm{~N} / \mathrm{m}$ (Novascan Technologies, Inc.) for fixed cells. 


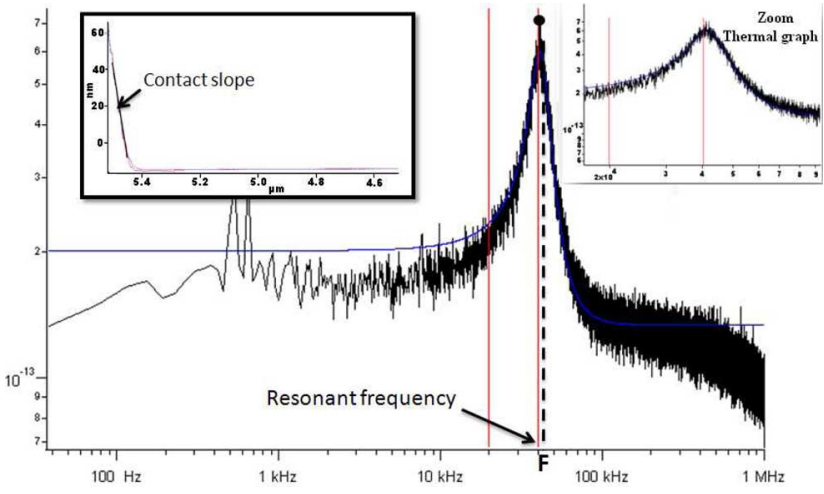

Fig. 8. Thermal graph measurement of a spherical tip cantilever $\left(K_{c}=\right.$ $1.29 \mathrm{~N} / \mathrm{m}$ ) used for fixed cells indentation. The left corner inset represents the contact slope from a force curve to determine the sensitivity of the cantilever and (deflection volt $=98.01 \mathrm{~nm} / \mathrm{v}$ ) and the right corner inset represents the zoom-in view of the resonant frequency plot $(F=46.043 \mathrm{kHz})$.

\section{B. AFM Calibration}

Before any experiment is done, it is necessary to perform a calibration of the cantilever to determine its resonant frequency. The resonant frequency measurement enables the user to determine if it lies within the specified range provided by the manufacturer in order to detect probe defects. The determination of spring constant $K_{n}$ by the thermal method involves both measurements : 1) sensitivity in nanometer per volt and 2) first-resonant frequency of the cantilever.

1) Determine the contact slope of the indentation curve on a hard surface to determine the sensitivity of the cantilever (see left corner inset of Fig. 8).

2) Measure the thermal power spectral density (PSD) to determine the resonant frequency of the cantilever and to confirm that the cantilever and light source are aligned. This confirms that the system is functioning properly. The results are plotted as the deflection amplitude data using Fourier transform in meter per square root hertz versus the frequency in hertz (see right corner inset of Fig. 8). The spring constant of the cantilever was determined experimentally for each tip used in our studies using the IGOR software interface supplied by Asylum Research.

Finally, the exact $K_{c}$ values after the calibration procedure are $K_{c}=0.08745 \mathrm{~N} / \mathrm{m}$ for live cells and $K_{c}=1.29 \mathrm{~N} / \mathrm{m}$ for fixed cells.

\section{MESC CHARACTERIZATION}

\section{A. Cell Culture Preparation}

It is recommended to use specially coated dishes or slides to facilitate adhesion. Such petri dishes or slides are available commercially. Cells should be attached to some rigid substrate, usually either a slide or the bottom of a petri dish. In our study, the mESC R1 (SCRC-1011, American Type Culture Collection (ATCC), Manassas, VA) were grown on $0.1 \%$ gelatin-coated plates in the absence of feeder cells. The ES medium consisted of $1000 \mathrm{U} / \mathrm{mL}$ leukemia inhibitory factor (LIF, ESGRO, Chemicon, Temecula, CA), 15\% fetal bovine serum (FBS) (Invitro-

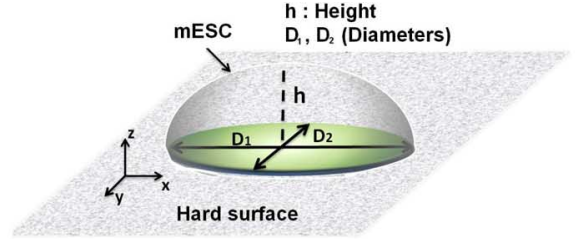

(a)

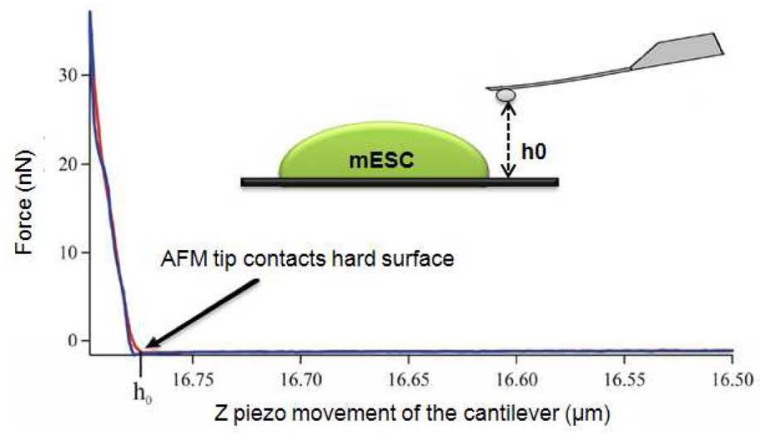

(b)

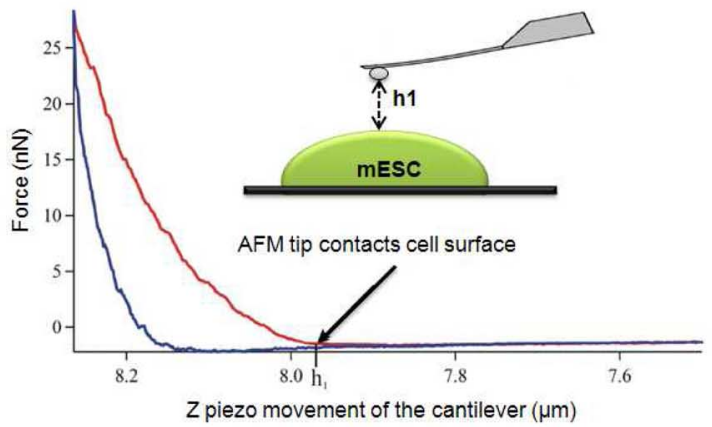

(c)

Fig. 9. How to determine the height of the stem cell through indentation curves? (a) mESC geometric parameters. (b) Force $(F)$ versus piezo movement of the cantilever $(Z)$ on a hard surface. The red and blue lines represent the loading and unloading curve, respectively. (c) Force $(F)$ versus piezo movement of the cantilever $(Z)$ on an mESC surface.

gen), and basic medium that included Knockout Dulbecco's modified Eagle's medium (Invitrogen), $2 \mathrm{mM}$ L-glutamine, 1x nonessential amino acids, and $0.1 \mathrm{mM}$ mercaptoethanol. Differentiation was induced by removal of LIF from the medium. Prior to the experiments, cells were dispersed using trypsin to obtain single cells and were plated on $60 \mathrm{~mm}$ tissue culture petri dish. Fixed mESC were obtained by treating live cells with with $4 \%$ formaldehyde for $10 \mathrm{~min}$, washed and stored in phosphate buffered saline.

\section{B. Geometry of the Cell and Cantilever Interaction With the Surface}

The cells were determined to be 10 and $15 \mu \mathrm{m}$ in diameter 368 using the cantilever tip to measure the interaction force between 369 the cell and the tip. The geometric modeling of the cell re- 370 quires the determination of the height, adhesion surface, and 371 cell's contour [see Fig. 9(a)]. The mESC height was calculated 372 from the force-displacement curve obtained on hard and mESC 373 surfaces. First, the initial height $h_{0}$ is measured by bringing 374 


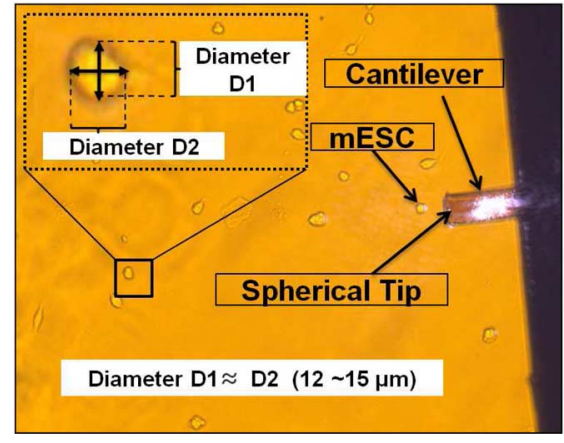

Fig. 10. How to determine the diameter of the stem cell through image analysis (ImagePro software from Asylum)?

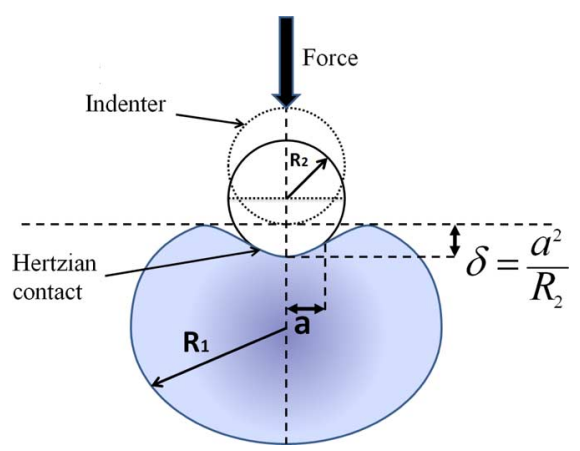

Fig. 11. Hertzian contact with a spherical tip: $F$ is the loading force, and $\delta$ and $a$ are the indentation and contact radius, respectively. the AFM tip in contact with the hard surface near mESC [see Fig. 9(b)], and then, the second height $h_{1}$ by bringing the AFM tip on mESC surface [see Fig. 9(c)]. The mESC height is the difference $h=h_{0}-h_{1}$. Second, to determine the mESC shape, AFM imaging experiments are carried out in order to determine the mESC's contour and diameter using image processing techniques [19]. From Fig. 10, we selected mESC shapes with circular adhesion surfaces. As illustration, the left corner inset shows a typical circular mESC, where $D_{1}$ and $D_{2}$ stands for the horizontal cells diameters ( $D_{1} \simeq D_{2}$ close to $12 \mu \mathrm{m}$ ).

\section{Mechanical Characterization of mESC Using Spherical Tip}

To estimate the mechanical properties of biological cells using the AFM, various analytical models can be used to identify the Young's modulus of mESC in live as well as fixed cells.

1) Hertz Contact: The Hertz contact model has been used extensively by the AFM community to quantify the mechanical property of biological samples using AFM [8], [9]. The Hertz contact model describes the simple case of elastic deformation of two perfectly homogeneous smooth surfaces touching under load (see Fig. 11). The forces measured are dominated by the elastic properties. In our work, the geometry of the tip used for all experiments is spherical. The mechanical interaction between the spherical tip and mESC can be described by the Hertz contact model of two elastic bodies [20]. The model assumes that:

1) the material properties of the tip and the cell are isotropic and homogeneous;

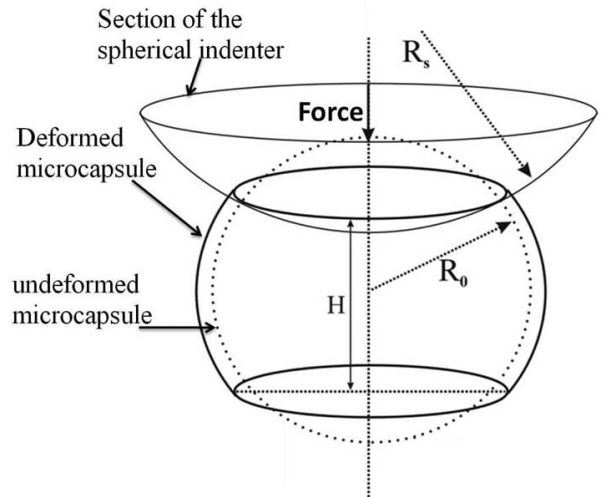

Fig. 12. Schematic of the microcapsule indented by a sphere with a loading force $F$.

2) the normal contact of the two bodies is adhesionless and 401 frictionless;

3) the contact geometry is assumed to be axisymmetric, smooth, and continuous.

The relationship between the indentation $\delta$, and the loading force $F$ is given by

$$
F=\frac{4}{3} E^{*} R^{\frac{1}{2}} \delta^{\frac{3}{2}}
$$

where $E^{*}$ and $R$ are the combined modulus and the relative curvature of the tip and the cell, respectively,

$$
\begin{aligned}
\frac{1}{E^{*}} & =\frac{1-\nu_{1}^{2}}{E_{1}}+\frac{1-\nu_{2}^{2}}{E_{2}} \\
\frac{1}{R} & =\frac{1}{R_{1}}+\frac{1}{R_{2}}
\end{aligned}
$$

where $\left(E_{1}, \nu_{1}, R_{1}\right)$ and $\left(E_{2}, \nu_{2}, R_{2}\right)$ represents the elastic modulus, Poisson's ratio, and the radius of the cell and spherical indenter, respectively. The elastic modulus of the silicon nitride cantilever and silicon cantilever were 222.22 and $168.17 \mathrm{GPa}$ used for the live and fixed cells, respectively. The elastic modulus of the cells is in range of kilopascals [11]. Hence, our assumption that the tip used for probing is infinitely stiff $\left(E_{2} \gg E_{1}\right)$ compared to the cell and Hertz contact model is valid. Thus, (13) can be rewritten as follows:

$$
F=\frac{4 E_{1}}{3\left(1-\nu_{1}^{2}\right)} R^{\frac{1}{2}} \delta^{\frac{3}{2}}
$$

where $E_{1}$ and $\nu_{1}$ represent the elastic modulus and Poisson's ratio of the cell assuming $\nu_{1}=0.5$.

2) Capsule Contact: The second model considers the biological cell to be composed of a cell membrane and cytoplasm (see Fig. 12). It is the capsule model. The model assumes that the cell membrane is a thin film and that the inner cytoplasm provides a uniform hydrostatic pressure on the membrane [22] The model assumes the following.

1) The cell membrane is linearly elastic.

2) The deformation of the cell membrane is caused by 427 stretching and bending of the cell membrane.

3) The cell is free of initial membrane stress or residual stress. 429

4) The cell volume is constant. 


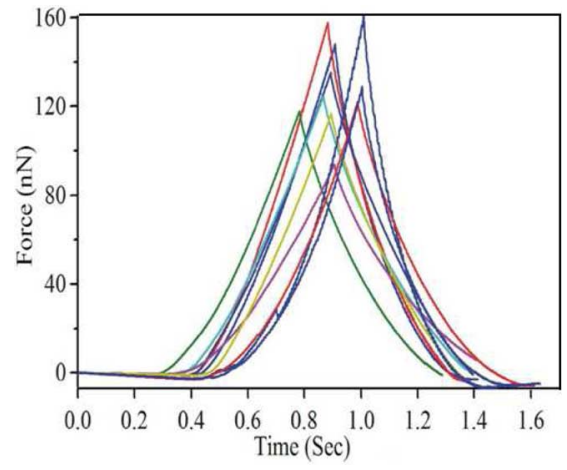

Fig. 13. Load-unload versus time for fixed undiff.

The force $(F)$ and relative deformation $(\varepsilon)$ relationship is given by [22]

$$
F=\frac{2 \pi h R_{0}\left[1+R_{0} /\left(2 R_{s}\right)\right]^{2} E}{(1-\nu)\left(1+R_{0} / R_{s}\right)^{4}} \varepsilon^{3}+\frac{\pi}{2 \sqrt{2}} E h^{2} \sqrt{\varepsilon}
$$

where $E, \nu$, and $h$ represent the elastic modulus, Poisson's ration, and the thickness of the capsule membrane, respectively. $R_{0}$ and $R_{s}$ are the radius of the capsule and the spherical indenter, respectively. $\varepsilon$ is the relative deformation of the capsule related to the indentation by $\varepsilon=\delta / 2 R_{0}$. The first and the second term in (17) represent stretching and bending of the cell membrane, respectively, however, in our experiments, the bending deformation term can be neglected for $\varepsilon>0.15$ [22].

\section{Comparison Between Experimental Data and Analytical Models}

We evaluated and compared the different analytical models to determine whether they appropriately predict the forceindentation relationship of mESC. Figs. 13 and 16 present the force (loading and unloading) versus time of fixed and live undiff mESC. From all these experiments, we observed adhesion force in few of the cases. For live mESC probed by a spherical tip, the adhesion force existed only for one sample. However, their amplitudes were negligible (less than 0.05 times the maximum indentation) compared to the maximum indentation forces (more than 0.1 times the maximum indentation) observed by other researchers [28], [29]. In our experiments, we observed that the adhesion force exists for only one of the live undiff and does not exist for the fixed cells. This could be due to the wear of the spherical tip [26]. Since we did not observe any adhesion forces, we have not used the JKR or DMT models.

Figs. 14 and 15 show the experimental force versus cell indentation curves for live and fixed undiff $\mathrm{mESC}$ and the simulated ones using the capsule and the Hertz models, respectively, for small and rather larger indentation range. Based on the experimental investigation, a least-square fit on the dataset (for the whole cells) and the corresponding $r^{2}$ values are calculated. These results show that the capsule model is inappropriate for describing $\mathrm{mESC}$ indentation. This conclusion is confirmed by the calculation of $r^{2}$ values performed on each mESC (see Table I). Finally, from our experiments, we infer that Hertz model appropriately describes the mechanical behavior of the

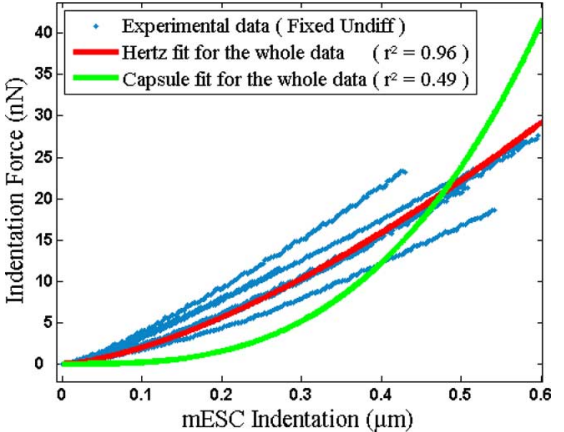

Fig. 14. Fixed undiff (small deformation).

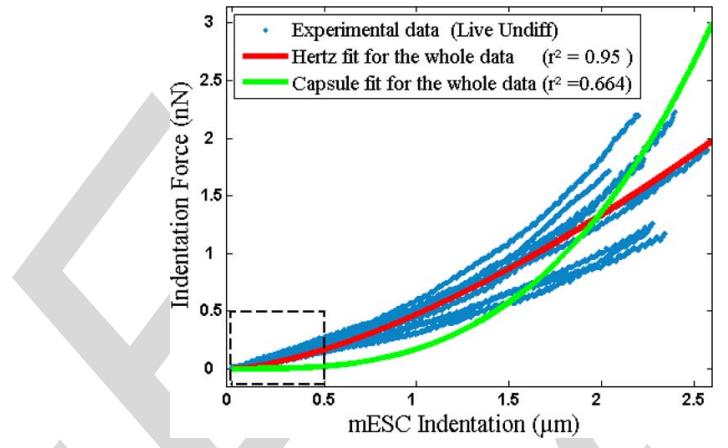

Fig. 15. Live undiff $\mathrm{mESC}$.

TABLE I

LeAST-SQUARE VAlue (COEFFICIENT CORRElation) $r^{2}$ OBTAINED By HeRTZ FIT AND CAPSUlE FIT FOR TEN SAMPLES OF LIVE AND FIXED UNDIF MESC

\begin{tabular}{|l|l|l|l|l|}
\cline { 2 - 5 } \multicolumn{1}{c|}{} & \multicolumn{2}{c|}{ Live Undiff mESC } & \multicolumn{2}{c|}{ Fixed Undiff mESC } \\
\cline { 2 - 5 } \multicolumn{1}{c|}{} & Hertz model & Capsule model & Hertz model & Capsule model \\
\hline Correlation $r^{2}$ & {$[0.98 \ldots . .99]$} & {$[0.68 \ldots . .0 .90]$} & {$[0.95 \ldots \mathbf{0 . 9 9}]$} & {$[0.46 \ldots . .0 .73]$} \\
\hline
\end{tabular}

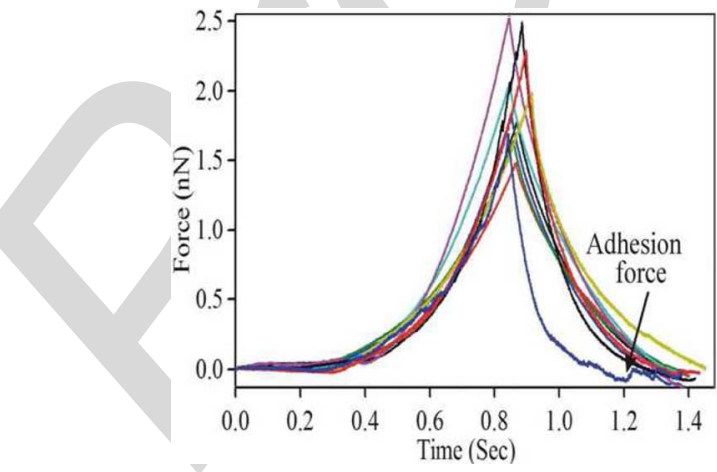

Fig. 16. Load-unload versus time for live undiff.

live and fixed undiff mESC (see Figs. 16- 18). Equation (16) was used to determine the global elastic modulus of the mESC. 470 The average elastic modulus were 17.87 and $0.217 \mathrm{kPa}$ for the 471 fixed and live undiff, respectively, (see Fig. 19). The standard 472 deviations for the fixed and live undiff cells were 3.37 and 473 $0.05 \mathrm{kPa}$. 


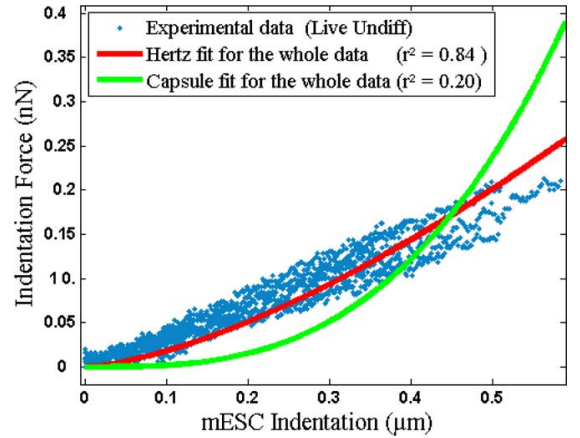

Fig. 17. Live undiff (small deformation).

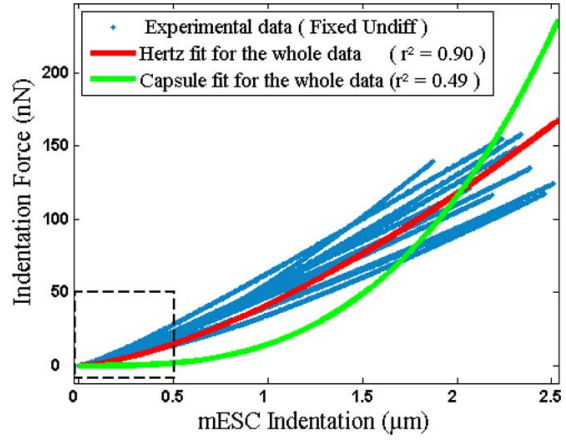

Fig. 18. Fixed undiff mESC.
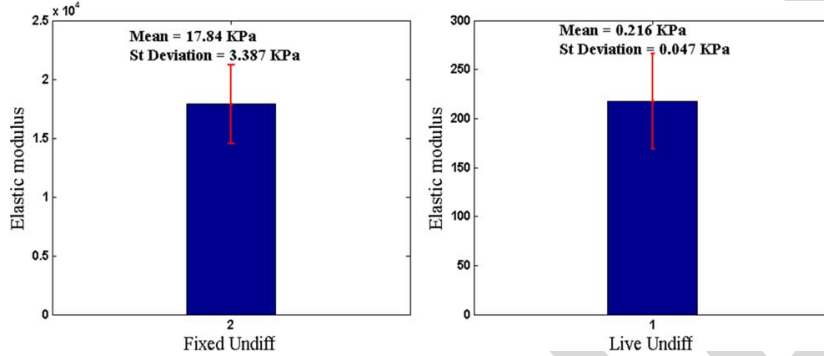

Fig. 19. Elastic modulus for live and fixed undifferentiated mESC calculated from the Hertz contact model for a small deformation (range $0.5 \mu \mathrm{m}$ ) with error bar.
It should be noticed that alterations in the mechanical properties of cells directly reflect changes in their cellular composition, internal structure (cytoskeleton), and external interactions (cellcell and/or cell-surface) that occur during differentiation, aging, and other changes in physiological status that are not accounted in the cell indentation model.

\section{FEM Simulation of CELl INDENTATION}

Fig. 20 presents a photo of the visual and haptic user interface setup. To test the accuracy and reliability of the proposed user interface system with haptics-enabled simulation, a set of experiments is designed using the setup given in Section II. The geometrical dimensions of the cell are determined through AFM image processing and force curve deflection (diameter and height), and the mechanical properties through AFM indentation studies due to the use of an analytical Hertz contact model.

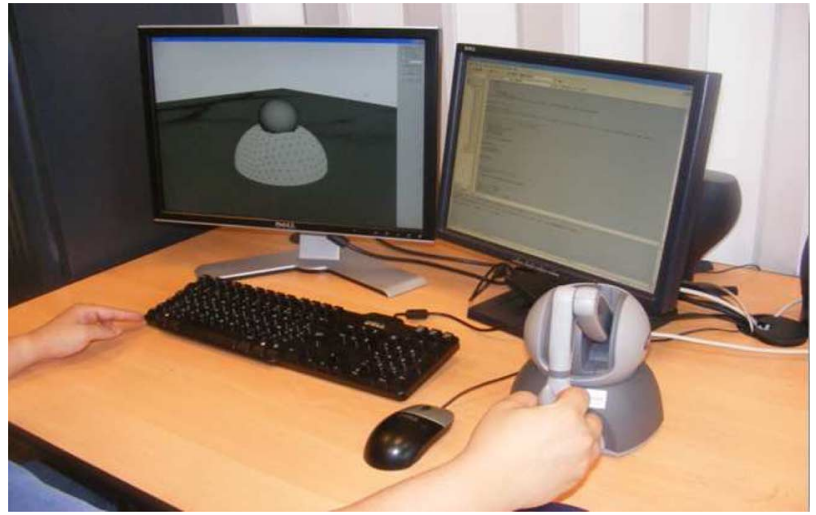

Fig. 20. Real-time indentation simulator using the Omni haptic interface.

TABLE II

Mechanical and Geometrical Properties of Single Cells Selected USED IN THE HAPTICS-ENABLED SIMULATOR

\begin{tabular}{c||cc}
\hline & Live mESC & Fixed mESC \\
\hline \hline Young modulus $(K P a)$ & 0.169 & 26.7 \\
Poisson coefficient & 0.49 & 0.49 \\
Diameter $(\mu \mathrm{m})$ & $\simeq 12$ & $\simeq 12$ \\
Height $(\mu \mathrm{m})$ & 8 & 8 \\
\hline
\end{tabular}

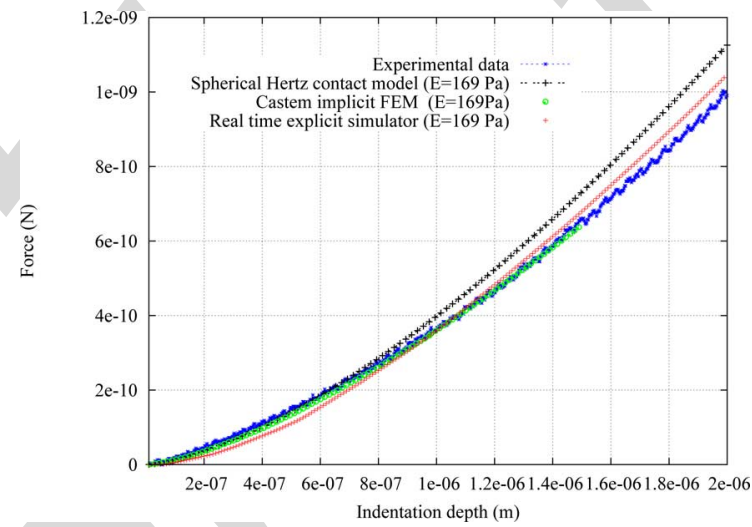

Fig. 21. Force versus indentation for live mESC: comparison between the real-time FEM, the Hertz contact, the quasi-static FEM, and experimental data.

These properties (see Table II) are passed to the finite-element 490 simulations.

In order to demonstrate the validity of our finite-element indentation model in real time, we compare the simulation indentation using the physics-based FEM model to the incremental data provided by AFM indentation tool and to the response simulated with a commercial FE software. Figs. 21 and 22 present the nonlinear relationship between the penetration distance (indentation) and the reaction force for live and fixed undiff cells. The finite-element simulations using the commercial software show good agreement with both Hertz contact model and the experimental indentation data. The developed real-time physicsbased FEM simulator demonstrates its accuracy in predicting 


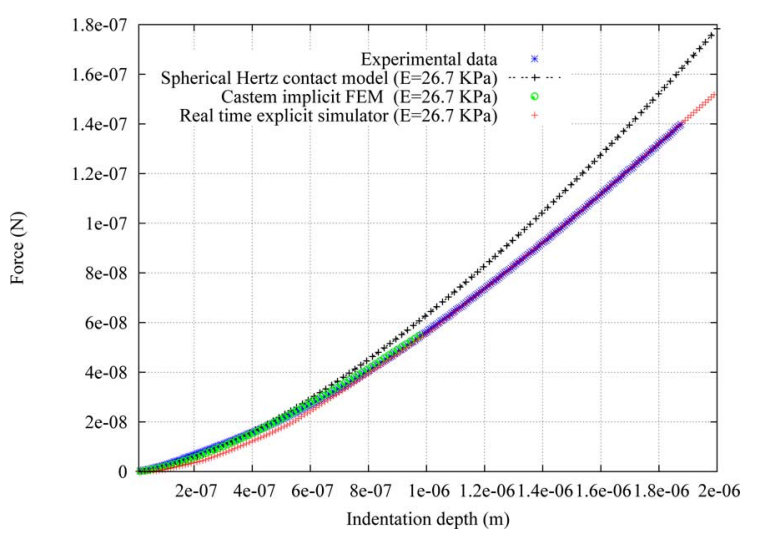

Fig. 22. Force versus indentation for fixed mESC: comparison between the real-time FEM, the Hertz contact, the quasi-static FEM, and experimental data.
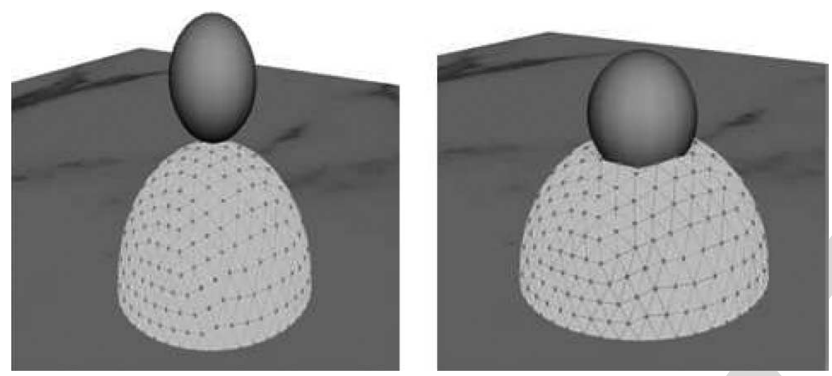

Fig. 23. Simulation of the AFM nanoindentation in VEs. The AFM tip and the stem cell was modeled using finite-element methods. A scene is shown from current training method: the 3-D cell deformation simulation before and after force indentation.

indentation forces for large deformations $(>25 \%)$ of relative nominal strain (see Figs. 21 and 22). Fig. 23 illustrates a realtime cell indentation procedure in a VE for training. It shows a screenshot of the cell surface deformation. The interaction between tip and surface can be visualized to the user in 3-D (sphere and cone represent AFM tip and forces acting on tip, respectively). It shows the indentation (elastic deformation) on the cell surface and repulsive contact forces while pressing on the surface. In order to improve the force feedback rendering, we added a visual metaphor on the computer screen.

The rendered reflection force is depicted by a cone representing the force vector acting on the AFM tip. The visual metaphor has efficiently proved its effectiveness in AFM-based nanomanipulation simulation [23]. The vector amplitude reflects the force feedback amplitude and the vector orientation represents the vertical/horizontal force components. Fig. 24 shows in 3-D real time the probe tip, zoomed cell indentation, and microforces. The indentation force rendered to the operator is composed of a vertical $F_{z}$ and horizontal $F_{x}$ component. For the specific case corresponding to the center indentation, the tangential component is very small $F_{x} \simeq 0$. Only vertical reflection force is provided to the user. He cannot haptically feel it during simulated indentation tests (see Figs. 25(a) and 26). In the case, where the AFM probe is pushed away from the center of the cell, a tangential component exists $\left(F_{x} \neq 0\right)$ and the reflec-

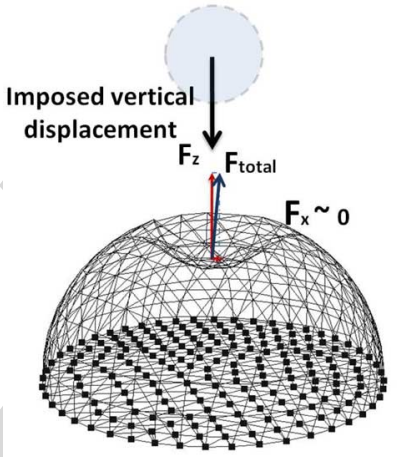

(a)

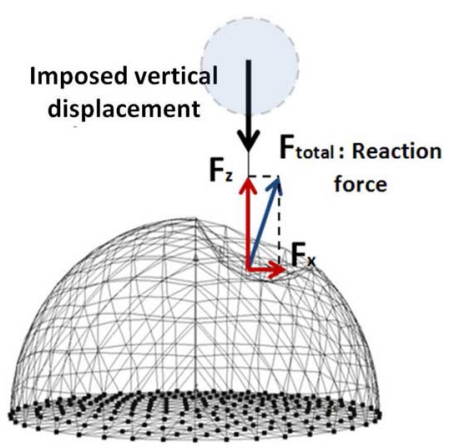

(b)
Fig. 25. Force indentation, where the probe is put (a) in the center and (b) off center of the cell.

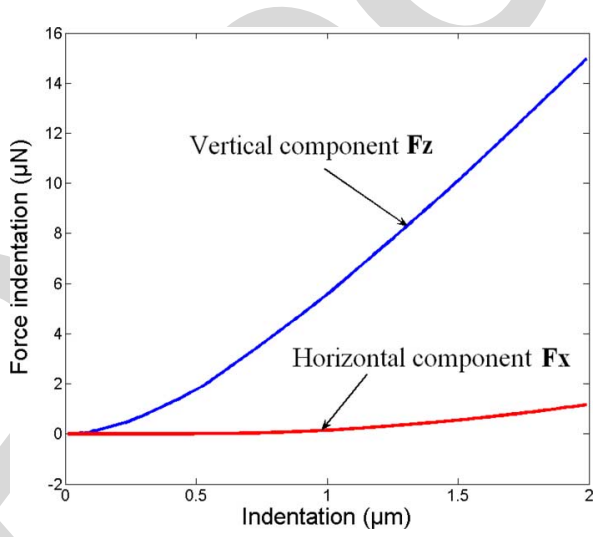

Fig. 26. Horizontal and vertical component force versus indentation: the probe is put in center of the cell.

tion force rendered to the user contains a vertical and tangential 528 component (see Figs. 25(b) and 27). We did not reproduce this 529 specific case in our results since the main interest of the paper is 530 to prove experimentally the real-time explicit simulator results 531 during centered cell indentations (since only vertical compo- 532 nents of the force experiments are measured through the AFM 533 cantilever). 


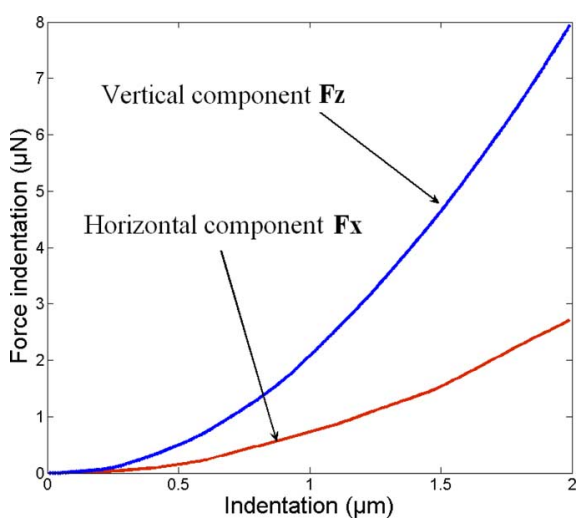

Fig. 27. Horizontal and vertical component force versus indentation: the probe is put off center of the cell.

\section{CONCLUSION}

We have developed a computer-based training system to simulate real-time cell indentation procedures in VEs for training biologists. The simulator provides the user with visual and haptic feedback. The simulation of this procedure involves real-time rendering of computer generated graphical images of the AFM tip, physics-based modeling of soft biological tissue, and display of touch and force sensations to the trainee through the simulation of haptic interactions. The real-time haptics-enabled simulator is realistic, since it is mainly based on experimental AFM data (mechanical and geometrical property of mESC) to accurately replicate the indentation task and predict the cell deformation during indentation. We first investigated the challenging issues in the real-time modeling of the biomechanical properties of the cell indentation through FEMs. Compared to experimental AFM indentation results performed on $\mathrm{mESC}$, we can see clearly that the proposed physically based FEM model is able to simulate the cell deformation through real-time simulation constraints. Currently, we are working on integrating nanoscale effects, such as friction, viscosity, punction, and adhesion forces in the nonlinear FEM model. All modalities will be merged in an ergonomic tool and intelligent biological simulator for stem cell characterization.

\section{REFERENCES}

[1] J. Settleman, "Tension precedes commitment-even for a stem cell," Mol. Cell, vol. 14, pp. 148-150, 2004.

[2] V. E. Meyers, M. Zayzafoon, J. T. Douglas, and J. M. McDonald, "RhoA and cytoskeletal disruption mediate reduced osteoblastogenesis and enhanced adipogenesis of human mesenchymal stem cells in modeled microgravity,” J. Bone Miner. Res., vol. 20, pp. 1858-1866, 2005.

[3] R. McBeath, D. M. Pirone, C. M Nelson, K. Bhadriraju, and C. S. Chen, "Cell shape, cytoskeletal tension, and RhoA regulate stem cell lineage commitment," Dev. Cell, vol. 6, pp. 483-495, 2004.

[4] J. Desai, A. Pillarisetti, and A. D. Brooks, "Engineering approaches to biomanipulation," Annu. Rev. Biomed. Eng., vol. 9, pp. 35-53, 2007.

[5] F. Arai, D. Ando, T. Fukuda, Y. Nonoda, and T. Oota, "Micromanipulation based on micro physics, strategy based on attractive force reduction and stress measurement," in Proc. IEEE Int. Conf. Robot. Autom., 1995, pp. 236-241.

[6] A. R. Bausch, W. Moller, and E. Sackmann, "Measurement of local viscoelasticity and forces in living cells by magnetic tweezers," Biophys. J., vol. 76, pp. 573-579, 1999.

[7] T. Ohasti, M. Hagiwara, D. L. Bader, and N. M. Knight, "Intracellular mechanics and mechanotransduction associated with chondrocyte defor- mation during pipette aspiration," Biorheology, vol. 43, no. 3-4, pp. 201214, 2006.

[8] J. L. Alonso and W. H. Goldmann, "Feeling the forces: atmoic force microscopy in cell biology," Life Sci., vol. 72, pp. 2553-2560, 2003.

[9] A. Touhami, B. Nysten, and Y. F. Dufrene, "Nanoscale mapping of the elasticity of microbial cells by atomic force microscopy," Langmuir, vol. 19, pp. 4539-4543, 2003.

[10] E. M. Darling, S. Zauscher, and F. Guilak, "Viscoelastic properties of zonal articular chondrocytes measured by atomic force microscopy," Osteoarthritis Cartilage, vol. 14, pp. 571-579, 2006.

[11] R. E. Mahaffy, S. Park, E. Gerde, J. Kas, and C. K. Shih, "Quantitative analysis of the viscoelastic properties of thin regions of fibroblasts using atomic force microscopy," Biophys. J., vol. 86, pp. 1777-1793, 2004.

[12] J. Alcaraz, L. Buscemi, M. Grabulosa, X. Trepat, B. Fabry, R. Farre, and D. Navajas, "Microrheology of human lung epithelial cells measured by atomic force microscopy," Biophys. J., vol. 84, pp. 2071-2079, 2004.

[13] A. Pillarisetti, C. Keefer, and J. P. Desai, "Mechanical Response of embryonic stem cells using haptics-enabled atomic force microscopy," in Proc. Int. Symp. Experimental Robot., Athens, Greece, 2008.

[14] R. E. Rudd, M. McElfresh, E. Baesu, R. Balhorn, M. Allen, and J. Belak, "Modeling of the Mechanical Deformation of Living Cells in Atomic force Microscopy," in Proc. Int. Conf. Comput. Nanosci., San Francisco, CA, 2003, pp. 23-27.

[15] V. Lulevich, T. Zink, H.-Y. Chen, F.-T. Liu, and G.-Y. Liu, "Cell mechanics using atomic force microscopy-based single-cell compression," Langmuir, vol. 22, no. 19, pp. 8151-8155, 2006.

[16] M. Bro-Nielsen and S. Cotin, "Real-time volumetric deformable models for surgery simulation using finite elements and condensation," Comput. Graph. Forum (Eurographics), vol. 5, no. 3, pp. 57-66, 1996.

[17] S. Cotin, H. Delingette, and N. Ayache, "A hybrid elastic model for realrime cutting, deformations, and force feedback for surgery training and simulation," Vis. Comput., vol. 16, pp. 437-452, 2000.

[18] J.-M. Schwartz, M. Dellinger, D. Rancourt, C. Moisan, and D. Laurendeau, "Modeling liver tissue properties using a non-linear visco-elastic model for surgery simulation," Med. Image Anal., vol. 9, no. 2, pp. 103-112, 2005.

[19] H. Ladjal, J.-L. Hanus, A. Pillarisetti, C. Keefer, A. Ferreira, and J. P Desai, "Atomic force microscopy-based single-cell indentation: Experimentation and finite element simulation," in Proc. IEEE Int. Conf. Robots Intell. Syst., St. Louis, MO, Oct. 11-15 2009.

[20] A. Pillarisetti, H. Ladjal, C. Keefer, A. Ferreira, and J. P. Desai, "Mechanical characterization of mouse embryonic stem cells," in Proc. 31st Ann Int. IEEE EMBS Conf., Minnesota, MN, Sep. 2-6 2009.

[21] A. Pillarisetti, C. Keefer, and J. P. Desai, "Mechanical response of embryonic stem cells using haptics-enabled atomic force microscopy," in Proc. Int. Symp. Exp. Robot., Athens, Greece, 2008.

[22] V. V. Lulevich, D. Andrienko, and O. I. Vinogradova, "Elasticity of polyelectrolyte multilayer microcapsules," J. Chem. Phys., vol. 120, no. 8, pp. 3822-3826, 2004.

[23] W. Vogl, K.-L.-Ma. Bernice, and M. Sitti, "Augmented reality user interface for an atomic force microscope-based nanorobotic system," IEEE Trans. Nanotechnol., vol. 5, no. 4, pp. 397-406, Jul. 2006.

[24] R. Adams, M. Moreyra, and B. Hannaford, "Stability and performance of haptic displays: Theory and experiments," in Proc. ASME Winter Annu. Meet. Haptics Workshop, Anaheim, CA, 1998.

[25] F. B. Llewellyn, "Some fundamental properties of transmission systems," Proc. IRE, vol. 40, 1952

[26] D. Maugis, Contact, Adhesion and Rupture of Elastic Solids, Springer 2000.

[27] H. Ladjal, J.-L. Hanus, A. Pillarisetti, C. Keefer, A. Ferreira, and J. P. Desai, "Realistic visual and haptic feedback simulator for real-time cell indentation," in Proc. IEEE Int. Conf. Robots Intell. Syst., Taipei, Taiwan, Oct. 18-22 2010.

[28] Y. Cao, D. Yang, and W. Soboyejoy, "Nanoindentation method for determining the initial contact and adhesion characteristics of soft polydimethylsiloxane," J. Mat. Res., vol. 20, no. 8, pp. 2004-2011, 2005.

[29] M. Girot, M. Boukallel, and S. Régnier, "Modeling soft contact mechanism of biological cells using an atomic bio-microscope," in Proc. Int Conf. Intell. Robots Syst., Beijing, China, 2006, pp. 1831-1836.

[30] E. U. Azeloglu and K. D. Costa, "Dynamic AFM elastography reveals phase dependent mechanical heterogeneity of beating cardiac myocyte," in Proc. IEEE Conf. Eng. Med. Biol. Soc., 2009, pp. 7180-7183.

[31] C. L. Kao, L. K. Tai, S. H. Chiou, Y. J. Chen, K. H. Lee, S. J. Chou, Y. L. Chang, C. M. Chang, S. J. Chen, H. H. Ku, and H. Y. Li, "Resveratrol 
promotes osteogenic differentiation and protects against dexamethasone damage in murine induced pluripotent stem cells," Stem Cells Dev., vol. 19, no. 2, pp. 247-258, Feb. 2010.

[32] H. Xie and S. Régnier, "Development of a flexible robotic system for multiscale applications of micro/nanoscale manipulation and assembly," IEEE/ASME Trans. Mechatronics, 2010.

[33] R. Perez, J. Agnus, C. Clevy, A. Hubert, and N. Chaillet, "Modeling, fabrication, and validation of a high-performance 2-DoF piezoactuator for micromanipulation," IEEE/ASME Trans. Mechatronics, vol. 10, no. 2, pp. 161-171, Apr. 2005.

[34] C. Elbuken, M. B. Khamesee, and M. Yavuz, "Design and implementation of a micromanipulation system using a magnetically levitated MEMS robot," IEEE/ASME Trans. Mechatronics, vol. 14, no. 4, pp. 434-445, Aug. 2009.

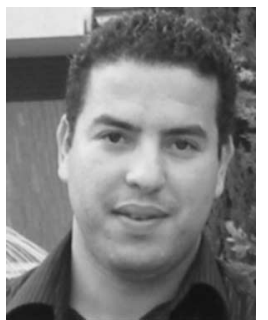

Hamid Ladjal received the Engineer degree in electronics and computing from Houari Boumediène University of Science and Technology, Algeria, in 2000, the Master's degree in virtual reality and complex systems from Evry-Val d'Essone University, Evry, France, and the Ph.D. degree in robotics from the University of Orléans, Orléans, France, in 2010.

$\mathrm{He}$ is currently an Associate Professor in the Institut PRISME, Ecole Nationale Supérieure d'Ingénieurs de Bourges, Bourges, France. His research interests include modeling and finite-element simulation, visual and haptic feedback, reality-based soft-tissue modeling for real-time simulation, image processing, control and micromanipulation, biological cell characterization, and cell biomechanics.

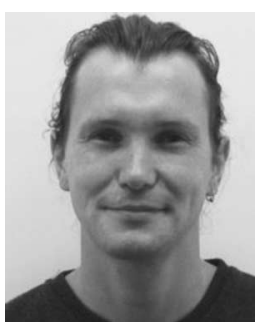

Jean-Luc Hanus received the M.S. degree from the National Aerospace and Mechanical Enginneering School, France, in 1993, and the Ph.D. degree in mechanical engineering from the University of Poitiers, Poitiers, France, in 1999.

He is currently an Assistant Professor of mechanical engineering at the Bourges National Engineering School, Bourges, France. His research interests include the area of nonlinear mechanical models and efficient numerical methods in dynamic analysis of biological cell responses.

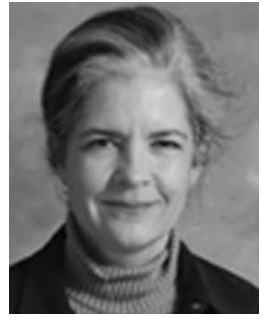

Carol Keefer is currently an Associate Professor in the Department of Animal and Avian Sciences, University of Maryland, College Park, MD, where she has established the new Biotechnology Program in Animal Sciences. Her laboratory explores mechanisms controlling development in mammalian embryos and embryonic stem cells and hopes to apply knowledge gained to the development of biomedical models of human and animal disease and to the production of therapeutic proteins via transgenic animals (biopharming). She was also a Senior Research Scientist at Nexia Biotechnologies Inc., Montreal, QC, Canada.

Prof. Keefer is a member of the Editorial Board of Cellular Reprogramming (formerly Cloning and Stem Cells). She is an active member of the Society for the Study of Reproduction (SSR). She was President of the International Embryo Transfer Society (IETS).

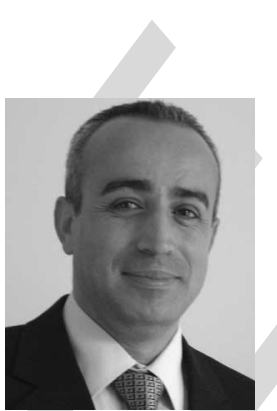

Antoine Ferreira (M'04) received the M.S. and $\mathrm{Ph} . \mathrm{D}$. degrees in electrical and electronics engineering from the University of Franche-Comté, Besancon, France, in 1993 and 1996, respectively.

In 1997, he was a Visiting Researcher in the ElectroTechnical Laboratory, Tsukuba, Japan. He is currently a Professor of robotics engineering at the Institut PRISME, Ecole Nationale Supérieure d'Ingénieurs de Bourges, Bourges, France. He is an author of three books on micro- and nanorobotics and more than 100 journal and conference papers and book contributions. His research interests include the design, modeling, and control of micro and nanorobotic systems using active materials, micronanomanipulation systems, biological nanosystems, and bionanorobotics.

Dr. Ferreira was the Guest Editor for different special issues of the IEEE/ASME TRANSACTIONS ON MECHATRONICS in 2009, International Journal of Robotics Research in 2009, and the IEEE Nanotechnology Magazine in 2008.

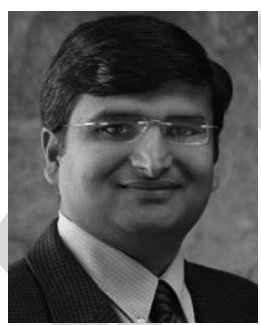

Jaydev P. Desai (SM'07) received the B.Tech. degree 744 from the Indian Institute of Technology, Bombay, 745 India, in 1993, and the M.A. degree in mathemat- 746 ics in 1997, and the M.S. and Ph.D. degrees in me- 747 chanical engineering and applied mechanics, in 1995748 and 1998, respectively, all from the University of 749 Pennsylvania, Philadelphia, PA.

He is currently an Associate Professor in the De- 751 partment of Mechanical Engineering, University of 752 Maryland, College Park, MD, where he is also the Di- 753 rector of the Robotics, Automation, and Medical Sys- 754 tems Laboratory. His research interests include image-guided surgical robotics, 755 haptics, reality-based soft-tissue modeling for surgical simulation, model-based 756 teleoperation in robot-assisted surgery, and cellular manipulation. $\quad 757$

Prof. Desai was the recipient of an NSF CAREER Award, a Lead Inventor 758 on the "Outstanding Invention of 2007 in Physical Science Category" at the 759 University of Maryland, and the Ralph R. Teetor Educational Award. He is 760 currently a member of the Haptics Symposium Committee, the Co-Chair of 761 the Surgical Robotics Technical Committee of the IEEE Robotics and Automa- 762 tion Society, and a member of the Editorial Board of the IEEE TRANSACTIONS 763 ON BIOMEDICAL ENGINEERING, ASME Journal of Medical Devices, and IEEE 764 TRANSACTIONS ON INFORMATION TECHNOLOGY IN BIOMEDICINE. He is also a 765 member of the American Society of Mechanical Engineers. 12 19 720 721 722

\section{5} 730 731 38
39 740 741 42 708
709 710 13 715 17 26 727
728 728
729 .

\section{.} (a) is

Anand Pillarisetti received the Undergraduate gree from the National Institute of Technology, Warangal, India, in 2002, the M.S. degree in mechanical engineering and mechanics from Drexel Unive in mechanical engineering from University of Maryland, College Park, MD, in 2008.

$\mathrm{He}$ is currently a Postdoctoral Researcher in the Department of Mechanical Engineering and Applied Mechanics, University of Pennsylvania, Philadelphia. His research interests include sensors, microelectromechanical systems, atomic force microscopy, and nanotechnology. 
Q1: Author: Please check and confirm footnote first as typeset.

Q2. Author: Please check and confirm the citation for Figs. 9 and 10 have been interchanged, with their respective captions, in 770 order to maintain sequential order in the text.

Q3. Author: Please spell out "JKR" and "DMT" in full, if possible.

Q4. Author: Please check and confirm in this sentence "Figs. 14 and 15 show......indentation range." the citations for Figs. 14 and 15 have been interchanged.

Q5. Author: Please check the citations for Figs. 16- 18 as typeset.

Q6. Author: Please provide page range for ref. [13], [19]-[21], [27].

Q7. Author: Please provide page range for Ref. [25].

Q8. Author: Please update Ref. [32].

Q9. Author: Please provide the title of Engineer and Master's degrees for H. Ladjal.

Q10. Author: Please provide the title of Undergraduate degree for A. Pillarisetti. 
- Reality-Based Real-Time Cell Indentation Simulator

\author{
Hamid Ladjal, Jean-Luc Hanus, Anand Pillarisetti, Carol Keefer, Antoine Ferreira, Member, IEEE, \\ and Jaydev P. Desai, Senior Member, IEEE
}

Abstract_-Training simulators that provide realistic visual and haptic feedback during cell indentation tasks are currently investigated. Complex cell geometry inherent to biological cells and intricate mechanical properties drive the need for precise mechanical and numerical modeling to assure accurate cell deformation and force calculations. Advances in alternative finite-element formulation, such as the mass-tensor approach, have reached a state, where they are applicable to model soft-cell deformation in real time. The geometrical characteristics and the mechanical properties of different cells are determined with atomic force microscopy (AFM) indentation. A real-time, haptics-enabled simulator for cell centered indentation has been developed, which utilizes the AFM data (mechanical and geometrical properties of embryonic stem cells) to accurately replicate the indentation task and predict the cell deformation during indentation in real time. This tool can be used as a mechanical marker to characterize the biological state of the cell. The operator is able to feel the change in the stiffness during cell deformation between fixed and live cells in real time. A comparative study with finite-element simulations using a commercial software and the experimental data demonstrate the effectiveness of the proposed physically based model.

Index Terms-Atomic force microscope (AFM), finite-element modeling (FEM), haptics, modeling, real-time interaction, stem cell.

Manuscript received March 31, 2010; revised July 7, 2010 and October 5, 2010; accepted October 8, 2010. Recommended by Technical Editor Y. Sun. A portion of this paper was presented at the IEEE/RSJ International Conference on Intelligent Robots and Systems, Taipei, Taiwan, October 18-22, 2010. This work was supported by the National Science Foundation under Grant CMMI 0826158, in part by STMD-Maryland Technology Development Corporation under Grant 08071517, and in part by the Centre de Recherche en Biologie de Baugy, France.

H. Ladjal, J.-L. Hanus, and A. Ferreira are with the Institut PRISME, Ecole Nationale Supérieure d'Ingénieurs de Bourges, 18000 Bourges, France (e-mail: hamid.ladjal@ensi-bourges.fr; jean-luc.hanus@ensi-bourges.fr; antoine.ferreira@ensi-bourges.fr).

A. Pillarisetti is with the Department of Mechanical Engineering and Applied Mechanics, University of Pennsylvania, Philadelphia, PA 19104 USA (e-mail :anandpi@ seas.upenn.edu.)

C. Keefer is with the Department of Animal and Avian Sciences, University of Maryland, College Park, MD 20742 USA (e-mail: ckeefer@umd.edu).

J. P. Desai is with the Robotics, Automation, and Medical Systems (RAMS) Laboratory, Maryland Robotics Center, Institute for Systems Research, University of Maryland, College Park, MD 20742 USA (e-mail: jaydev@umd.edu).

Color versions of one or more of the figures in this paper are available online at http://ieeexplore.ieee.org.

Digital Object Identifier 10.1109/TMECH.2010.2091010

\section{The Promise of Stem Cell Research}

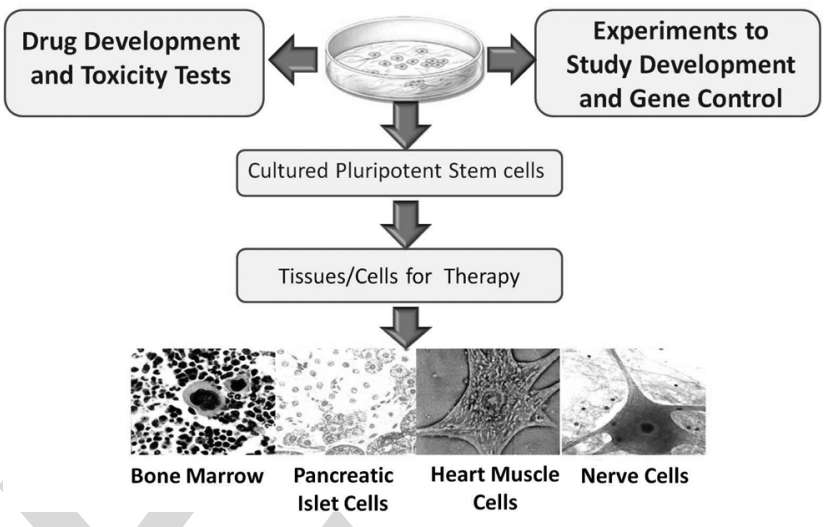

Fig. 1. Stem cells are valuable resources for toxicology, developmental biology, and regenerative medicine research.

important role in cell development and differentiation [1]-[3]. In contrast to adult stem cells, embryonic stem cells differentiate into every cell type in the body, and thus, have a huge therapeutic potential. Stem-cell-based systems offer a very promising and innovative solution for obtaining large number of cells for early efficacy and toxicity screening. Stem cell technology provides a new tool for drug development and a better insight to understand mammalian gene function (see Fig. 1). The mechanical properties of biological cells have been studied with different techniques [4], the most popular being optical tweezers [5], magnetic beads [6], and micropipette aspiration [7]. Different microrobotic systems with various end effectors have been proposed [33], [34]. However, these methods cannot compete with the precision that can be obtained with the atomic force microscopy (AFM) method [8], [32]. AFMs have been widely used in the study of micro- and nanostructures including living cells. Modern AFM techniques allow solving several problems of cell biomechanics by simultaneous evaluation of the local mechanical properties and the topography of living cells, at a high spatial resolution and force sensitivity [9]. In these experiments, an AFM cantilever serves as a microindenter to probe the cell, and further analysis of force-indentation data yields the local Young's modulus. In addition, the AFM indentation technique can be used to characterize the viscoelastic behavior of the cell cytoskeleton [10], including viscosity [11], loss and storage moduli [12], and stress relaxation times. Mechanical phenotyping prove actually to be a valuable tool in the development of improved methods of targeted cellular differentiation of embryonic, induced pluripotent (iPS), and adult stem cells for therapeutic and diagnostic purposes [30], [31]. In this study, we propose to characterize the mechanical behavior of an individual mouse embryonic stem cells (mESC) in undifferentiated state 
using AFM indentation [13]. As we hypothesize that the cell stiffness of mESC may reflect the nuclear physical changes of the cell, it should be possible to detect modifications in stiffness by probing the cell directly. However, different limitations are inherent in AFM-based indentation techniques. First, deformations induced by the AFM probe and cell positions cannot be seen by the user. Second, the force sensing of the AFM cannot resolve the force interaction between the AFM tip and the cell's surface independently in all three dimensions to avoid cytoskeleton-disrupted experiments. Therefore, the user performs extremely challenging manipulations requiring advanced manual skills. Modeling the physics of the interaction between tip and cell is necessary to realize complicated indentation tasks like cancer cell probing [14].

We developed finite-element models (FEMs) that include the topological information of adherent stem cells (shape and dimensions), AFM tips (conical and spherical), and biological structure (cell models with cytoplasm layers, cytoskeletons, and nucleus) [15]. To validate the viscoelastic and nonlinear dynamics of the cell in real time using finite-element procedures, we report herein the mechanical characterization of single mESC indentation-relaxation for different colonies of fixed and live undifferentiated mESCs (termed, undiff $m E S C$ ). The proposed indentation tests use microsphere-modified AFM probes in order to estimate the global elastic modulus of the cell to reflect the true global response of a mESC [14], [15]. This paper is a subsequent contribution to [27], where various analytical models are tested and compared to estimate the mechanical properties of the biological cells. Based on the proposed physical model, we have developed a computer-based training system using force feedback to simulate cell indentation procedures in virtual environments (VEs) for biologist training. The paper consists of five sections. In Section II, we present the biomechanical finite-element approach dedicated to real-time indentation. In Section III, we present the methodology of the VE system for cell indentation. In Section IV, we present the experimental setup using the force microscopy. In Section V, we make a comparative study between the physics-based model and experimental data. In Section VI, we present our real-time cell indentation simulator using a spherical tip. Finally, we give some concluding remarks and the directions for future work.

\section{Physics-Based Deformable Models for Cell NANOINDENTATION SIMULATION}

In this section, we present further developments to the linear elastic mass-tensor model introduced by [16], [17] and extended by Schwartz et al. [18]. In our paper, the cell is modeled by a volume object discretized into a conformal tetrahedral mesh as defined by finite-element theory.

Using classical notations, inside each tetrahedron $T^{k}$, the displacement field, defined by a linear interpolation $\left[\mathbf{N}^{\mathrm{k}}\right]$ of the nodal displacement vector $\left\{\mathbf{u}^{\mathrm{k}}\right\}$ of the four vertices of tetrahedron, is written as follows:

$$
\left\{\mathbf{U}(\mathbf{x})^{\mathbf{k}}\right\}=\left[\mathbf{N}^{\mathbf{k}}(\mathbf{x})\right] \quad\left\{\mathbf{u}^{\mathbf{k}}\right\}
$$

For a linear elastic material, the relation between the Cauchy 118 stress tensor and the linearized strain tensor is written with 119 Lame's coefficient in condensed vector notation as follows:

$$
\{\boldsymbol{\sigma}\}=\lambda\left(\{\varepsilon\}_{1}+\{\varepsilon\}_{2}+\{\varepsilon\}_{3}\right)[\mathbf{I}]+2 \mu\{\varepsilon\}
$$

where $[\mathbf{I}]$ is the identity matrix.

The principle of virtual work applied to a single tetrahadron $T^{k}$ leads to the elementary stiffness matrix $\left[\mathbf{K}^{\mathbf{k}}\right]$ such that the elementary nodal force vector acting on a tetrahedron is as follows:

$$
\left\{\mathbf{f}^{\mathrm{k}}\right\}=\left[\mathbf{K}^{\mathrm{k}}\right]\left\{\mathbf{u}^{\mathrm{k}}\right\} .
$$

This stiffness matrix is composed of a plurality of elementary submatrices, each connecting the elementary force acting on the node $i$ to the displacement of the node $j$

$$
\begin{aligned}
{\left[\mathbf{K}_{\mathbf{i j}}^{\mathbf{k}}\right]=\frac{1}{36 V^{k}} } & \left(\lambda\left\{\mathbf{m}_{\mathbf{i}}\right\}\left\{\mathbf{m}_{\mathbf{j}}\right\}^{T}\right. \\
& \left.+\mu\left\{\mathbf{m}_{\mathbf{j}}\right\}\left\{\mathbf{m}_{\mathbf{i}}\right\}^{T}+\mu\left\{\mathbf{m}_{\mathbf{i}}\right\}^{T}\left\{\mathbf{m}_{\mathbf{j}}\right\}[\mathbf{I}]\right)
\end{aligned}
$$

where $\{\mathbf{m}\}$ are unit outward-pointing normals to triangular faces and $V^{k}$ is the volume of the tetrahedron $T^{k}$.

Taking into account the contribution of all adjacent tetrahedra, the global internal force acting on a node $l$ can be expressed as follows:

$$
\left\{\mathbf{F}_{\mathbf{i n t}}^{\mathbf{l}}\right\}=\sum_{k \in \mathcal{V}_{l}}\left(\sum_{j=1}^{4}\left[\mathbf{K}_{\mathbf{i j}}^{\mathbf{k}}\right]\left\{\mathbf{u}_{\mathbf{j}}\right\}\right)
$$

where $\mathcal{V}_{l}$ is the neighborhood of vertex $l$ (i.e., the tetrahedra containing node $l$ ).

The tensors $\left[\mathbf{K}_{\mathrm{ij}}^{1}\right]$ depending on the remaining geometry and Lame's coefficients are constant. They can be precomputed in an offline phase. This is the essential advantage of the mass-tensor approach, which makes it useful for real-time application.

\section{A. Cell Modeling Using FEM}

In our paper, the mESC is meshed with 3-D first-order tetrahedral elements, as shown in Fig. 2. The geometrical characteristics of the cells were obtained from experimental data using the AFM system. This includes shape, diameter, and height of the cells. The mesh that has been used for the following simulations is composed of 835 vertices and 2456 tetrahedra, where all vertices are free to move except the ones in contact with the petri dish, which are fixed.

\section{B. Dynamic Model}

The equation of motion of a vertex $l$ of the cell mesh can be written as follows:

$$
M^{l}\left\{\ddot{\mathbf{u}}_{l}\right\}+\gamma^{l}\left\{\dot{\mathbf{u}}_{l}\right\}+\sum_{k \in \mathcal{V}_{l}}\left(\sum_{j=1}^{4}\left[\mathbf{K}_{\mathbf{i j}}^{\mathbf{k}}\right]\left\{\mathbf{u}_{\mathbf{j}}\right\}\right)=\left\{\mathbf{F}_{\text {ext }}^{\mathbf{l}}\right\}
$$

where $M^{l}$ and $\gamma^{l}$ are, respectively, the mass and damping coefficients of each vertex. 


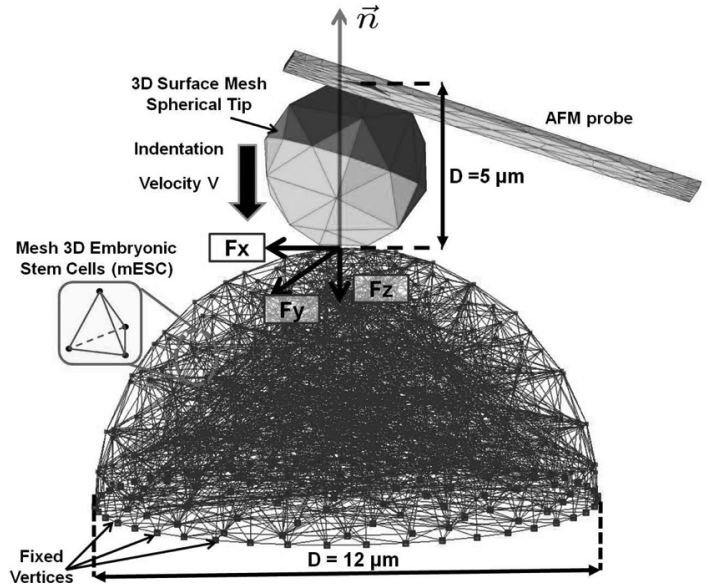

Fig. 2. Representation of the 3-D mESC mesh using P1 tetrahedral finite elements.

To solve the dynamic system, we tested different integration schemes (implicit and explicit) taking into account the tradeoff between real-time simulation and haptic stability requirements. We choose the explicit centered finite-difference scheme defined by

$$
\left\{\begin{array}{l}
u(t+h)=u(t)+\dot{u}(t) h+\frac{1}{2} \ddot{u}(t) h^{2}+O\left(h^{3}\right) \\
\dot{u}(t+h)=\dot{u}(t)+\frac{1}{2} \ddot{u}(t) h+\frac{1}{2} \ddot{u}(t+h) h+O\left(h^{3}\right)
\end{array}\right.
$$

where $h$ is the temporal integration step size chosen to satisfy the Courant-Friedrich-Lewy condition.

The discrete equation of motion of a vertex $l$ reads

$$
\begin{aligned}
\left(M^{l}+\frac{h}{2} \gamma^{l}\right)\left\{\ddot{\mathbf{u}}_{l}\right\}_{(t+h)} \\
=\left\{\mathbf{F}_{\mathbf{e x t}}^{\mathbf{l}}\right\}_{(t+h)}-\gamma^{l}\left(\left\{\dot{\mathbf{u}}_{l}\right\}_{(t)}+\frac{h}{2}\left\{\ddot{\mathbf{u}}_{l}\right\}_{(t)}\right) \\
\quad-\sum_{k \in \mathcal{V}_{l}}\left(\sum_{j=1}^{4}\left[\mathbf{K}_{\mathbf{i j}}^{\mathbf{k}}\right]\left(\left\{\mathbf{u}_{\mathbf{j}}\right\}_{(t)}+h\left\{\dot{\mathbf{u}}_{j}\right\}_{(t)}+\frac{h^{2}}{2}\left\{\ddot{\mathbf{u}}_{j}\right\}_{(t)}\right)\right)
\end{aligned}
$$

from which we can compute the new vertices for accelerations, velocities, and positions.

\section{VE SYSTEM FOR CELL INDENTATION}

Fig. 3 shows the architecture of the real-time virtual-realitybased cell indentation simulator. This includes the computer generated models of stem cells, the AFM nanoindentor, the collision detection algorithm, physics-based models of deformable $\mathrm{mESC}$, and the haptic interaction controller. The operator will be able to interact with the 3-D model of the cell using his sense of vision as well as actively manipulate using his sense of touch. Usually, real-time graphics translates to an update rate of $25 \mathrm{~Hz}$, stable haptic interaction in VE's requires much higher update rate of around $1 \mathrm{kHz}$. As soft biological tissues exhibit complex viscoelastic and nonlinear properties, the realtime interaction imposes severe restrictions on the FEM model.

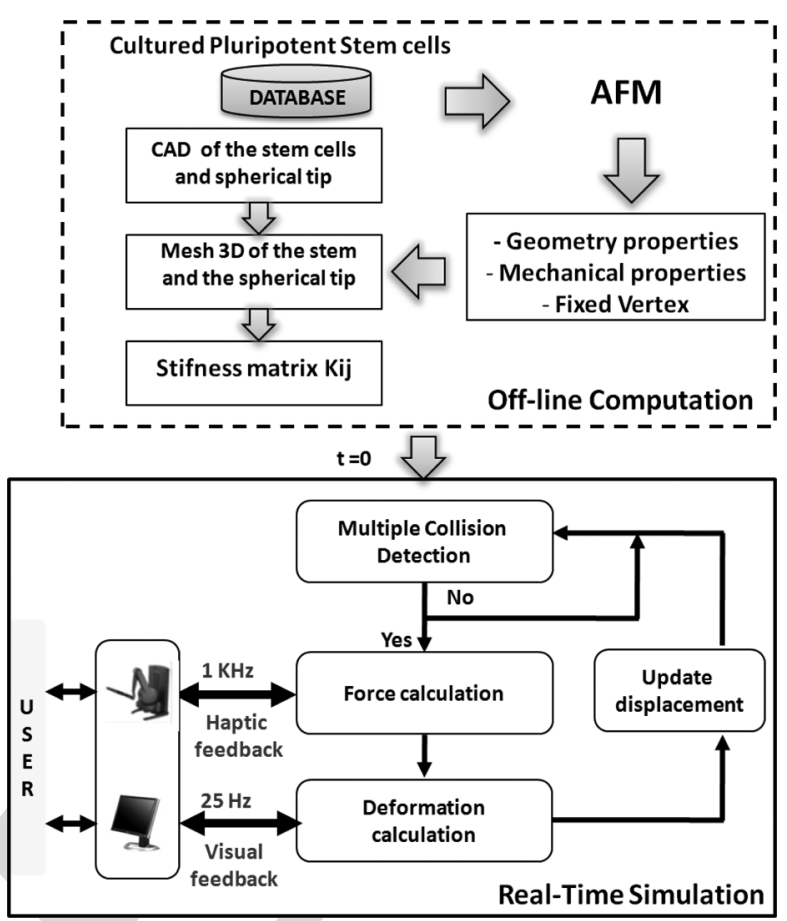

Fig. 3. Computational architecture for simulating force-reflecting deformable cell indentation in a VE. The figure shows the two simulation phases used for the real-time indentation of the cell: 1) offline precalculations of stiffness matrices and 2) simulation of visual and haptic interaction.

A significant difficulty of using the finite-element technique for 177 real-time simulation is that it is computationally costly. These 178 mesh-based schemes also require an expensive numerical in- 179 tegration operation for the computation of the system stiffness 180 matrices. For this reason, we adopted a computational architec- 181 ture with two simulation stages: 1) an offline computation of 182 stiffness matrices for each triangular element and 2) visual and 183 haptic interaction for real-time indentation simulation.

\section{A. Offline Computation}

The proposed computational methodology contains an offline precalculation step. The most costly and time-consuming operations are realized during this step. The database contains various developed models of the different stem cells geometry (bone marrow, pancreatic, heart muscle, nerve cells, etc.). Using AFM image analysis (ImagePro software from AFM Asylum), we derived different geometric stem cell models based on a commercial computer-aided design (CAD) package (MARC-ADAMS). The meshing of the 3-D internal structure of the mESC is then carried out through a dedicated 3-D meshing software (GID software) modeled in exact dimensions. Although they were displayed as 3-D texture-mapped objects to the user, they were modeled as connected line segments to reduce the number of collision computations during real-time interactions.

The adherent cell was approximated as an assembly of discrete tetrahedral elements interconnected to each other through a fixed number of nodes (see Fig. 2). The displacements of these nodal points for applied external indentation forces were the basic unknowns of our FEM analysis. The coordinates 


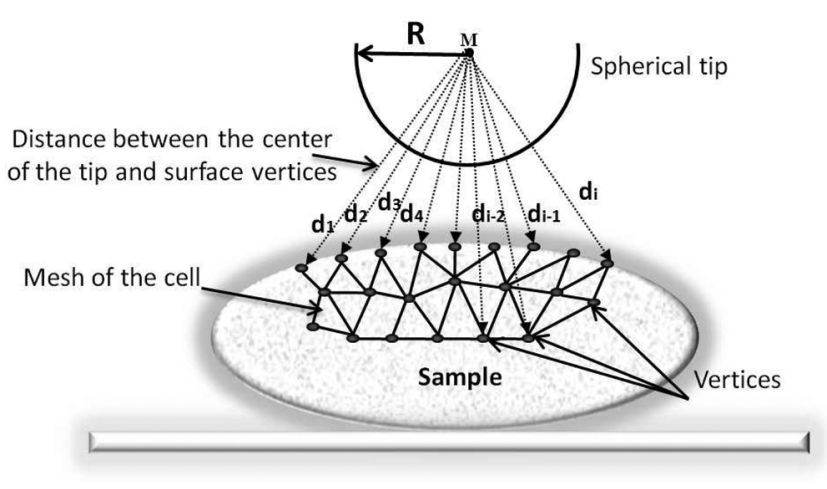

Fig. 4. Scheme illustrating the multiple collision detection algorithm using a simple sphere-points intersection problem: the distance between the center of the spherical tip and the nodes of the cell surface is calculated in real time.

of vertices, the tetrahedron indexing, and the connectivity of vertices were derived from the geometric model. Then, the mechanical (Young's modulus and stiffness) and geometrical (height and shape) properties of the cell structure are determined through AFM indentation experiments. Finally, these properties are passed to the FEM algorithm for calculating the physically based behavior of biological soft tissues. The offline calculation of all tensors $K_{i j}$ allows precalculation stiffness values for preselected cells such as, for example, live and fixed cells.

\section{B. Real-Time Indentation}

Since haptics and graphics have different update frequencies, we implemented separate threads to update the loops. During the indentation task, the contact between the tip and the cell must occur at a special set of points (nodal points).

1) Collision Detection: An important part of biological cantilever tip-soft cell modeling is the fast collision detection algorithm. The collision detection is the first step to carry out realistic interactions. However, locating the contact primitive (e.g., facets) between two objects may be computationally expensive, especially if the objects are composed of a large number of polygons. In general, to determine a contact between two virtual objects, we compute the distance between them. If this distance is negative, then the objects are in contact. We may continue by determining the set of geometric objects or entities that collide. The cantilever tip in VE can be modeled as a complex 3-D object, composed of numerous surfaces, edges, and vertices, but this constitutes a challenging task. For the purpose of detecting collisions, we used a point-based representation of the cantilever tip as a simple spherical object and employed a simple sphere-points intersection algorithm with local search technique. It calculates the distance between the center of the spherical tip and the nodes of the cell surface (see Fig. 4).

2) Penalty-Based Models: In an interaction model, a collision between two objects may generate local and global deformations of the colliding objects, new internal forces, changes in the velocities of the involved objects, and topology modifications, such as punction or cutting. Physical simulations suppose that these events are related to the mechanical properties of the objects. In our simulations, we used the penalty-based models.

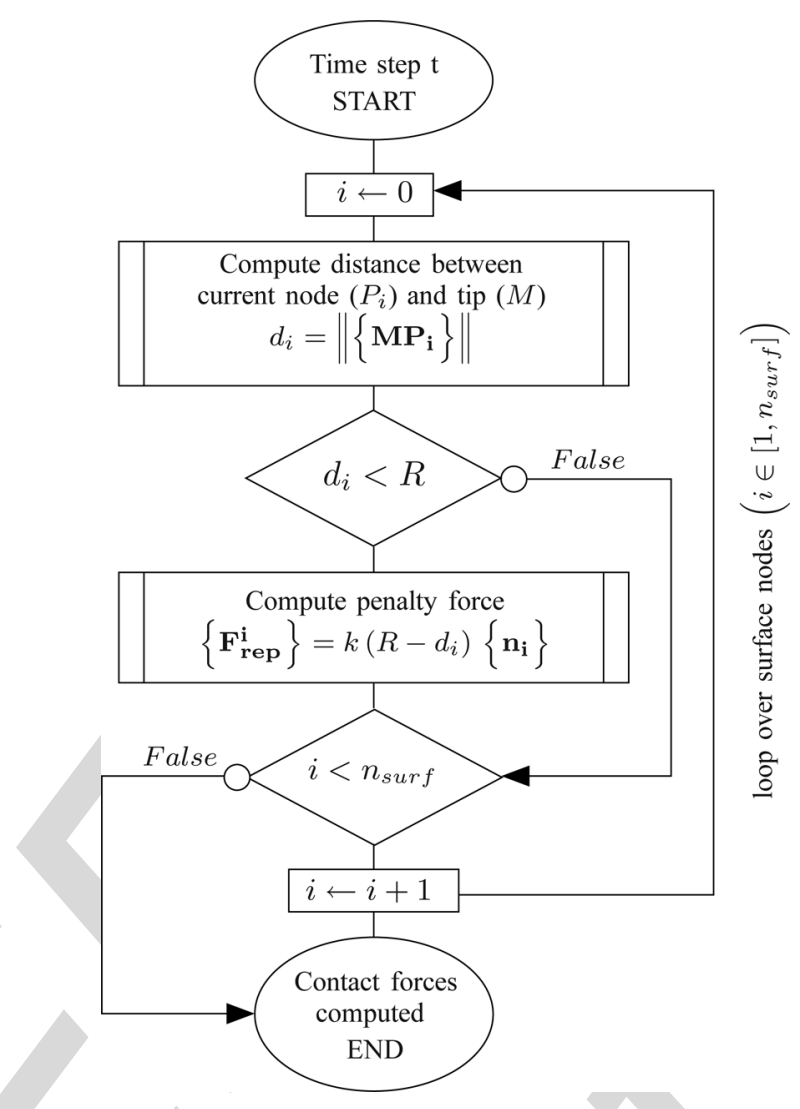

Fig. 5. Main flowchart for multi collision detection: $M$ represents the position of the center of the spherical tip and $R$ its radius, $i$ represents the current external cell surface node, $d_{i},\left\{F_{\text {rep }}^{i}\right\}$ and $\left\{n_{i}\right\}$ represent, respectively, the computed distance between the center of the tip and the current node, the repulsive force, and the outward-pointing contact normal at node $i$.

These models consider the reactive force that first reduces the 244 relative speed of the two objects, and finally, repulses them from 245 each other, as proportional to the deformation. Thus, the reac- 246 tive force is a function of the local interpenetration depth $\delta$. The 247 normal repulsive force $F_{\text {rep }}$ can be written as follows:

$$
\left\{F_{\text {rep }}\right\}=\lambda \delta\{n\}
$$

where $\lambda$ is the penalty of the interpenetration and $\{n\}$ is the 249 outward-pointing normal.

These contact forces are evaluated at each time step $t$ (see Fig. 5). They represent the new updated external forces acting upon our stem cell.

3) Virtual Coupling for Stability of the Haptic Rendering: The internal operating loop of the haptic interface requires an update frequency around $1 \mathrm{kHz}$. However, the FEM update frequency is in the range of $25-30 \mathrm{~Hz}$. This frequency difference threatens the coherence between both systems leading to instabilities of the user haptic rendering. We adopted the solution to use a virtual coupling model defined in [24].

This approach introduces a virtual passive link between the simulation model and the haptic interface in order to ensure the stability and the performance of the system [see Fig. 6(a)]. When we combine the impedance display implementation with an appropriate virtual coupling network, we get the admittance 


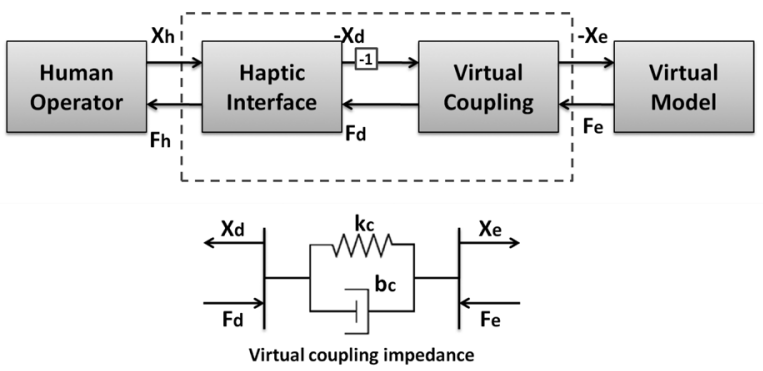

(a)

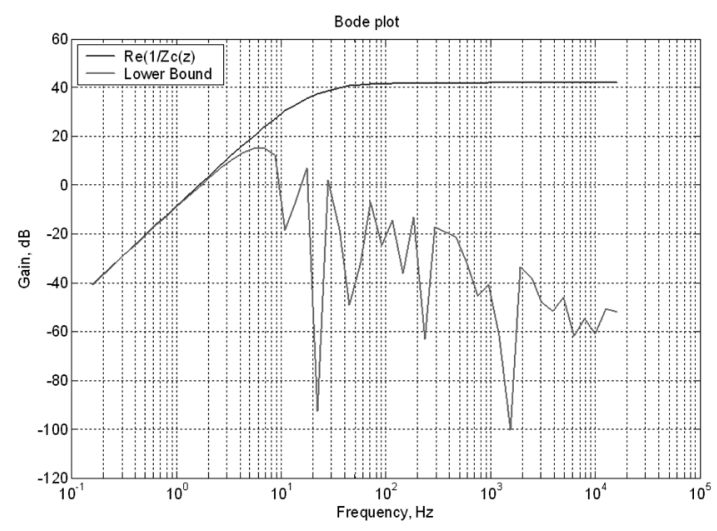

(b)

Fig. 6. Stability conditions using the virtual coupling model. (a) Model of virtual coupling. (b) Impedance display virtual coupling.

matrix for the combined interface. The linear two-port is said to be absolutely stable if there exists no set of passive terminating one-port impedances for which the system is unstable. Llewellyn's stability criteria [25] provides both necessary and sufficient conditions for absolute stability of linear twoports. We get the conditions for absolute stability of the haptic interface

$$
\operatorname{Re}\left(Z_{\mathrm{di}}(z)\right) \geq 0, \frac{1}{Z_{\mathrm{cvi}}(z)} \geq 0
$$

$$
\cos (\angle \mathrm{ZOH}(z))+\frac{2 \operatorname{Re}\left(Z_{\mathrm{di}}(z)\right) \operatorname{Re}\left(1 / Z_{\mathrm{cvi}}(z)\right)}{|\mathrm{ZOH}(z)|} \geq 1
$$

where $Z_{\mathrm{cvi}}(z)$ is the virtual coupling impedance $\left(k_{c}, b_{c}\right)$, $\mathrm{ZOH}(z)$ is a zero-order holder, and $Z_{\mathrm{di}}(z)$ is the PHANToM impedance. The inequality (11) can be rewritten to get an explicit expression of absolute stability of the haptic interface

$$
\operatorname{Re}\left(\frac{1}{Z_{\mathrm{cvi}}(z)}\right) \geq \frac{1-\cos (\angle \mathrm{ZOH}(z))}{2 \operatorname{Re}\left(Z_{\mathrm{di}}(z)\right)}|\mathrm{ZOH}(z)| .
$$

For the virtual coupling, the impedance display induces a limit on the maximum impedance which can be rendered. We use (12) to find the virtual coupling, which makes the haptic interface absolutely stable. Using the design bound of the best performing, absolutely stabilizing virtual coupling. These parameters are found as $b_{c}=0.008 \mathrm{~N} /\left(\mathrm{mm} / \mathrm{s}\right.$ ) and $k_{c}=2.3 \mathrm{~N} / \mathrm{mm}$ (plotted on Fig. 6(a) as a thin red line). The left side of (12) with the resulting values is plotted on Fig. 6(b) as a bold blue line.

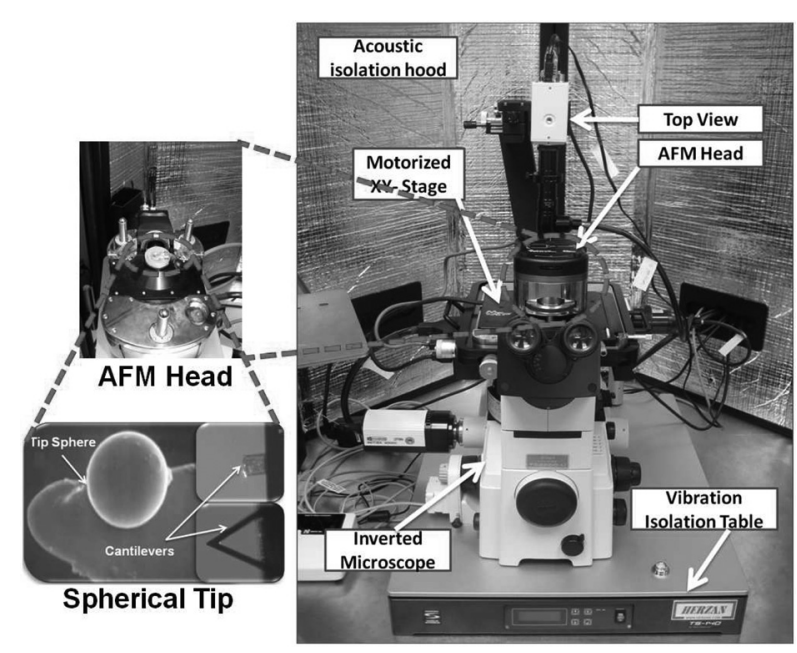

Fig. 7. Experimental setup based on AFM system for mESC indentation studies. The nanoindentor is constituted by a spherical probe $(5 \mu \mathrm{m}$ diameter $)$ attached to a silicon nitride cantilever from Novascan Technologies.

\section{EXPERIMENTAL SETUP}

\section{A. Description}

The experimental tests of the cell indentations were performed using an AFM (Model: MFP-3D-BIO, Asylum Research, Santa Barbara, CA). The AFM is integrated with a top view module and mounted on an active vibration isolation table manufactured by Herzan (Laguna Hills, CA), as shown in Fig. 7. The top view module enables viewing of cells and easy alignment of the laser beam on the AFM cantilever. The XY stage (manual) allows the user to position the cell beneath the cantilever tip of the AFM. The entire AFM setup is enclosed in an acoustic isolation chamber to prevent acoustic noise from interfering with the AFM measurements. The $x$ - and $y$-axis ranges of the scan head are $90 \mu \mathrm{m}$. The $z$-axis scan range is $40 \mu \mathrm{m}$. The AFM system is used to obtain force and cell deformation data from biological samples.

The cantilever is moved by the piezoelectric scanner in $z$ direction toward the cell. The deflection of the cantilever is detected by a photodiode when the tip comes in contact with the cell. When the tip of the cantilever is in contact with the cell, the initial cantilever deflection $\left(d_{0}\right)$, and initial cantilever movement in $(z)$ direction $\left(z_{0}\right)$ are stored. As the cantilever moves further in $z$-direction and deforms the cell, the final cantilever deflection $\left(d_{1}\right)$ and the cantilever movement $\left(z_{1}\right)$ are obtained [21].

The stiffness depends not only on the Young's modulus but also on the geometry of the tip-surface contact. Therefore, the geometry and spring constant of the cantilever are calibrated in the same way for live and fixed cells. In the following experiments, we employed two types of cantilevers attached with a spherical probe ( $5 \mu \mathrm{m}$ in diameter).

1) Silicon nitride cantilever with a spring constant of 0.06 $\mathrm{N} / \mathrm{m}$ (Novascan Technologies, Inc., Ames, IA) for live cells.

2) Silicon cantilever with a spring constant of $1.75 \mathrm{~N} / \mathrm{m}$ (Novascan Technologies, Inc.) for fixed cells. 


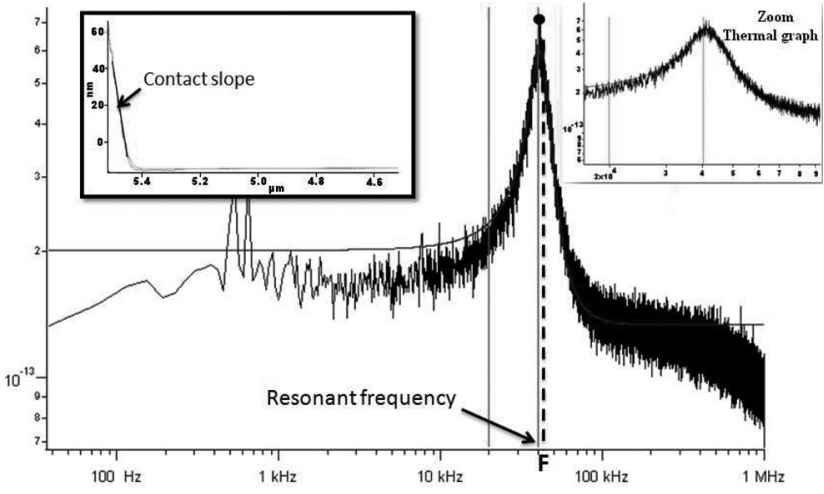

Fig. 8. Thermal graph measurement of a spherical tip cantilever $\left(K_{c}=\right.$ $1.29 \mathrm{~N} / \mathrm{m}$ ) used for fixed cells indentation. The left corner inset represents the contact slope from a force curve to determine the sensitivity of the cantilever and (deflection volt $=98.01 \mathrm{~nm} / \mathrm{v}$ ) and the right corner inset represents the zoom-in view of the resonant frequency plot $(F=46.043 \mathrm{kHz})$.

\section{B. AFM Calibration}

Before any experiment is done, it is necessary to perform a calibration of the cantilever to determine its resonant frequency. The resonant frequency measurement enables the user to determine if it lies within the specified range provided by the manufacturer in order to detect probe defects. The determination of spring constant $K_{n}$ by the thermal method involves both measurements : 1) sensitivity in nanometer per volt and 2) first-resonant frequency of the cantilever.

1) Determine the contact slope of the indentation curve on a hard surface to determine the sensitivity of the cantilever (see left corner inset of Fig. 8).

2) Measure the thermal power spectral density (PSD) to determine the resonant frequency of the cantilever and to confirm that the cantilever and light source are aligned. This confirms that the system is functioning properly. The results are plotted as the deflection amplitude data using Fourier transform in meter per square root hertz versus the frequency in hertz (see right corner inset of Fig. 8). The spring constant of the cantilever was determined experimentally for each tip used in our studies using the IGOR software interface supplied by Asylum Research.

Finally, the exact $K_{c}$ values after the calibration procedure are $K_{c}=0.08745 \mathrm{~N} / \mathrm{m}$ for live cells and $K_{c}=1.29 \mathrm{~N} / \mathrm{m}$ for fixed cells.

\section{MESC CHARACTERIZATION}

\section{A. Cell Culture Preparation}

It is recommended to use specially coated dishes or slides to facilitate adhesion. Such petri dishes or slides are available commercially. Cells should be attached to some rigid substrate, usually either a slide or the bottom of a petri dish. In our study, the mESC R1 (SCRC-1011, American Type Culture Collection (ATCC), Manassas, VA) were grown on $0.1 \%$ gelatin-coated plates in the absence of feeder cells. The ES medium consisted of $1000 \mathrm{U} / \mathrm{mL}$ leukemia inhibitory factor (LIF, ESGRO, Chemicon, Temecula, CA), 15\% fetal bovine serum (FBS) (Invitro-

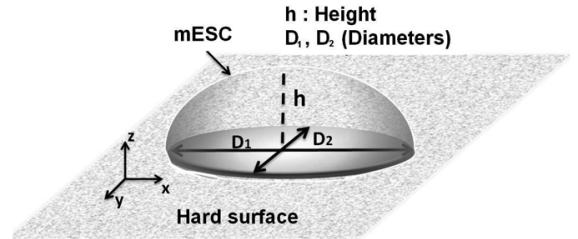

(a)

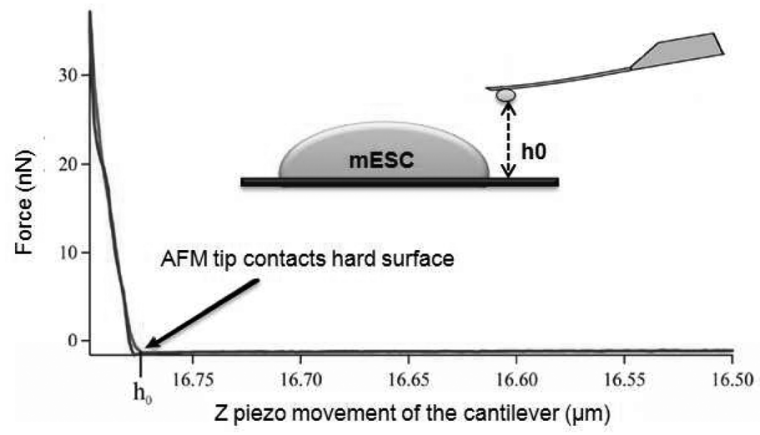

(b)

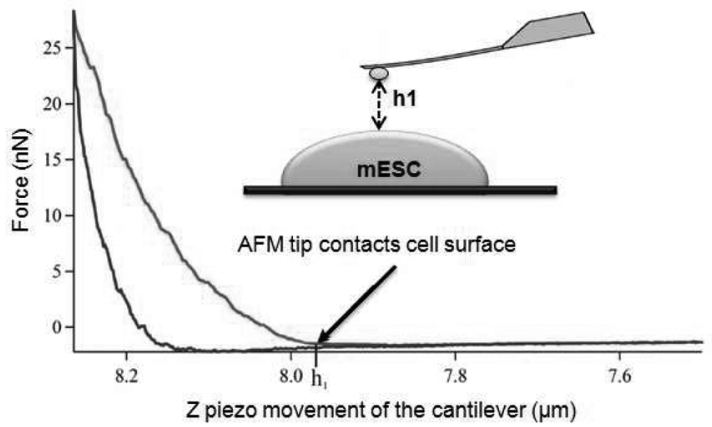

(c)

Fig. 9. How to determine the height of the stem cell through indentation curves? (a) mESC geometric parameters. (b) Force $(F)$ versus piezo movement of the cantilever $(Z)$ on a hard surface. The red and blue lines represent the loading and unloading curve, respectively. (c) Force $(F)$ versus piezo movement of the cantilever $(Z)$ on an mESC surface.

gen), and basic medium that included Knockout Dulbecco's 357 modified Eagle's medium (Invitrogen), 2 mM L-glutamine, 1x 358 nonessential amino acids, and $0.1 \mathrm{mM}$ mercaptoethanol. Dif- 359 ferentiation was induced by removal of LIF from the medium. 360 Prior to the experiments, cells were dispersed using trypsin to 361 obtain single cells and were plated on $60 \mathrm{~mm}$ tissue culture petri 362 dish. Fixed mESC were obtained by treating live cells with with 363 $4 \%$ formaldehyde for $10 \mathrm{~min}$, washed and stored in phosphate 364 buffered saline.

\section{B. Geometry of the Cell and Cantilever Interaction With the Surface}

The cells were determined to be 10 and $15 \mu \mathrm{m}$ in diameter 368 using the cantilever tip to measure the interaction force between 369 the cell and the tip. The geometric modeling of the cell re- 370 quires the determination of the height, adhesion surface, and 371 cell's contour [see Fig. 9(a)]. The mESC height was calculated 372 from the force-displacement curve obtained on hard and mESC 373 surfaces. First, the initial height $h_{0}$ is measured by bringing 374 


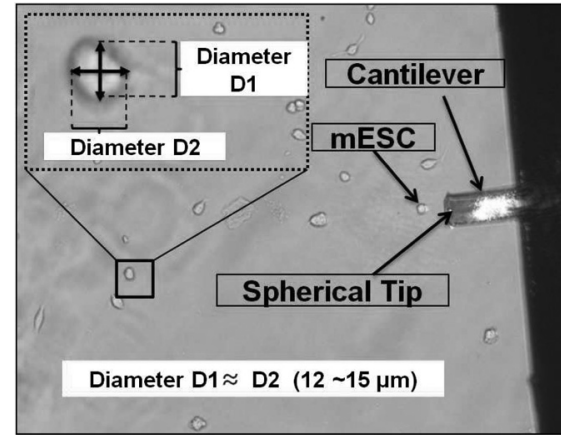

Fig. 10. How to determine the diameter of the stem cell through image analysis (ImagePro software from Asylum)?

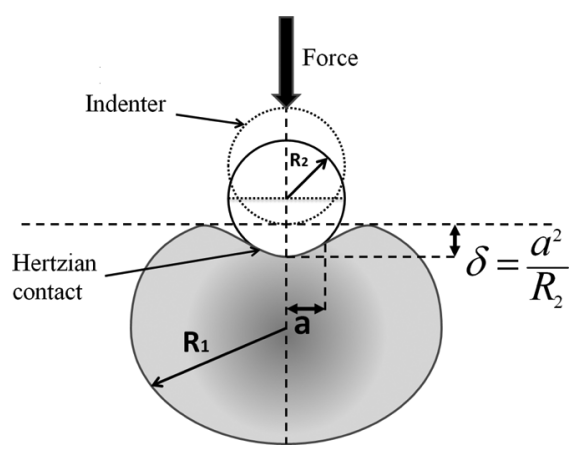

Fig. 11. Hertzian contact with a spherical tip: $F$ is the loading force, and $\delta$ and $a$ are the indentation and contact radius, respectively. the AFM tip in contact with the hard surface near mESC [see Fig. 9(b)], and then, the second height $h_{1}$ by bringing the AFM tip on mESC surface [see Fig. 9(c)]. The mESC height is the difference $h=h_{0}-h_{1}$. Second, to determine the mESC shape, AFM imaging experiments are carried out in order to determine the mESC's contour and diameter using image processing techniques [19]. From Fig. 10, we selected mESC shapes with circular adhesion surfaces. As illustration, the left corner inset shows a typical circular mESC, where $D_{1}$ and $D_{2}$ stands for the horizontal cells diameters ( $D_{1} \simeq D_{2}$ close to $12 \mu \mathrm{m}$ ).

\section{Mechanical Characterization of mESC Using Spherical Tip}

To estimate the mechanical properties of biological cells using the AFM, various analytical models can be used to identify the Young's modulus of mESC in live as well as fixed cells.

1) Hertz Contact: The Hertz contact model has been used extensively by the AFM community to quantify the mechanical property of biological samples using AFM [8], [9]. The Hertz contact model describes the simple case of elastic deformation of two perfectly homogeneous smooth surfaces touching under load (see Fig. 11). The forces measured are dominated by the elastic properties. In our work, the geometry of the tip used for all experiments is spherical. The mechanical interaction between the spherical tip and mESC can be described by the Hertz contact model of two elastic bodies [20]. The model assumes that:

1) the material properties of the tip and the cell are isotropic and homogeneous;

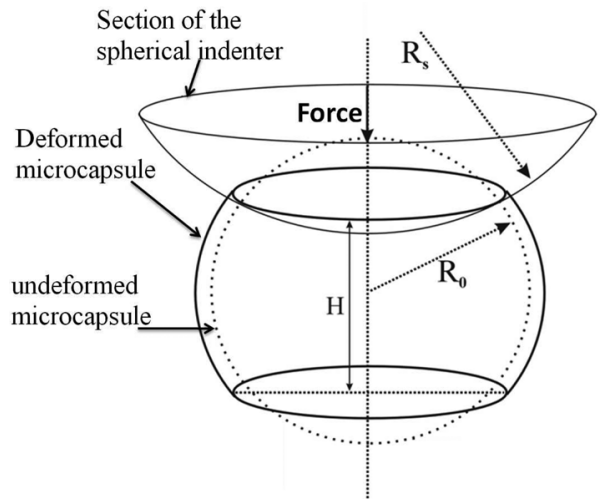

Fig. 12. Schematic of the microcapsule indented by a sphere with a loading force $F$.

2) the normal contact of the two bodies is adhesionless and 401 frictionless;

3) the contact geometry is assumed to be axisymmetric, smooth, and continuous.

The relationship between the indentation $\delta$, and the loading force $F$ is given by

$$
F=\frac{4}{3} E^{*} R^{\frac{1}{2}} \delta^{\frac{3}{2}}
$$

where $E^{*}$ and $R$ are the combined modulus and the relative curvature of the tip and the cell, respectively,

$$
\begin{aligned}
\frac{1}{E^{*}} & =\frac{1-\nu_{1}^{2}}{E_{1}}+\frac{1-\nu_{2}^{2}}{E_{2}} \\
\frac{1}{R} & =\frac{1}{R_{1}}+\frac{1}{R_{2}}
\end{aligned}
$$

where $\left(E_{1}, \nu_{1}, R_{1}\right)$ and $\left(E_{2}, \nu_{2}, R_{2}\right)$ represents the elastic modulus, Poisson's ratio, and the radius of the cell and spherical indenter, respectively. The elastic modulus of the silicon nitride cantilever and silicon cantilever were 222.22 and $168.17 \mathrm{GPa}$ used for the live and fixed cells, respectively. The elastic modulus of the cells is in range of kilopascals [11]. Hence, our assumption that the tip used for probing is infinitely stiff $\left(E_{2} \gg E_{1}\right)$ compared to the cell and Hertz contact model is valid. Thus, (13) can be rewritten as follows:

$$
F=\frac{4 E_{1}}{3\left(1-\nu_{1}^{2}\right)} R^{\frac{1}{2}} \delta^{\frac{3}{2}}
$$

where $E_{1}$ and $\nu_{1}$ represent the elastic modulus and Poisson's ratio of the cell assuming $\nu_{1}=0.5$.

2) Capsule Contact: The second model considers the biological cell to be composed of a cell membrane and cytoplasm (see Fig. 12). It is the capsule model. The model assumes that the cell membrane is a thin film and that the inner cytoplasm provides a uniform hydrostatic pressure on the membrane [22] The model assumes the following.

1) The cell membrane is linearly elastic.

2) The deformation of the cell membrane is caused by stretching and bending of the cell membrane.

3) The cell is free of initial membrane stress or residual stress. 429

4) The cell volume is constant. 


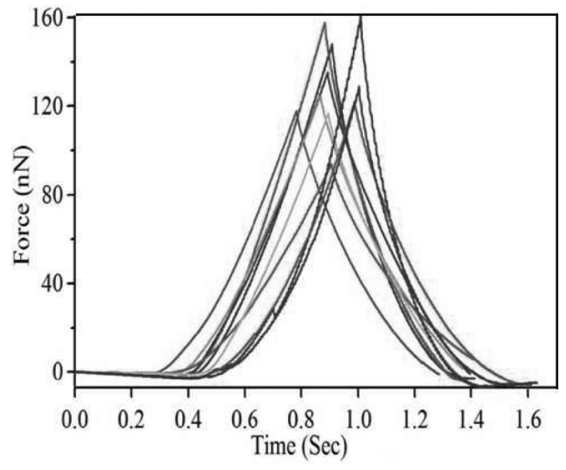

Fig. 13. Load-unload versus time for fixed undiff.

The force $(F)$ and relative deformation $(\varepsilon)$ relationship is given by [22]

$$
F=\frac{2 \pi h R_{0}\left[1+R_{0} /\left(2 R_{s}\right)\right]^{2} E}{(1-\nu)\left(1+R_{0} / R_{s}\right)^{4}} \varepsilon^{3}+\frac{\pi}{2 \sqrt{2}} E h^{2} \sqrt{\varepsilon}
$$

where $E, \nu$, and $h$ represent the elastic modulus, Poisson's ration, and the thickness of the capsule membrane, respectively. $R_{0}$ and $R_{s}$ are the radius of the capsule and the spherical indenter, respectively. $\varepsilon$ is the relative deformation of the capsule related to the indentation by $\varepsilon=\delta / 2 R_{0}$. The first and the second term in (17) represent stretching and bending of the cell membrane, respectively, however, in our experiments, the bending deformation term can be neglected for $\varepsilon>0.15$ [22].

\section{Comparison Between Experimental Data and Analytical Models}

We evaluated and compared the different analytical models to determine whether they appropriately predict the forceindentation relationship of mESC. Figs. 13 and 16 present the force (loading and unloading) versus time of fixed and live undiff mESC. From all these experiments, we observed adhesion force in few of the cases. For live mESC probed by a spherical tip, the adhesion force existed only for one sample. However, their amplitudes were negligible (less than 0.05 times the maximum indentation) compared to the maximum indentation forces (more than 0.1 times the maximum indentation) observed by other researchers [28], [29]. In our experiments, we observed that the adhesion force exists for only one of the live undiff and does not exist for the fixed cells. This could be due to the wear of the spherical tip [26]. Since we did not observe any adhesion forces, we have not used the JKR or DMT models.

Figs. 14 and 15 show the experimental force versus cell indentation curves for live and fixed undiff $\mathrm{mESC}$ and the simulated ones using the capsule and the Hertz models, respectively, for small and rather larger indentation range. Based on the experimental investigation, a least-square fit on the dataset (for the whole cells) and the corresponding $r^{2}$ values are calculated. These results show that the capsule model is inappropriate for describing $\mathrm{mESC}$ indentation. This conclusion is confirmed by the calculation of $r^{2}$ values performed on each mESC (see Table I). Finally, from our experiments, we infer that Hertz model appropriately describes the mechanical behavior of the

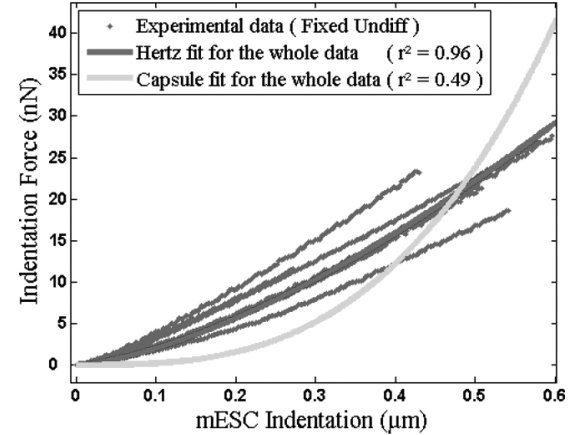

Fig. 14. Fixed undiff (small deformation).

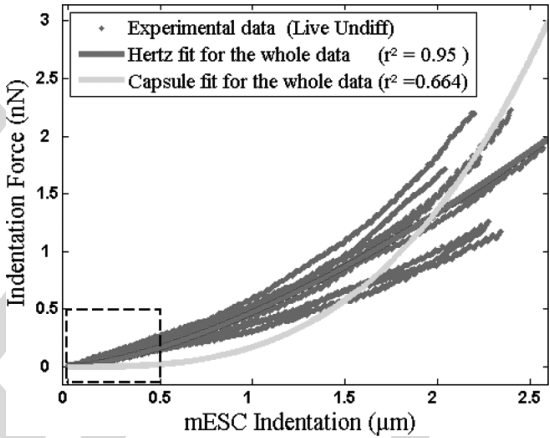

Fig. 15. Live undiff mESC .

TABLE I

LeAST-SQUARE VAlue (COEFFICIENT CORRElation) $r^{2}$ OBTAINED By HeRTZ FIT AND CAPSULE FIT FOR TEN SAMPLES OF LIVE AND FIXED UNDIF MESC

\begin{tabular}{|l|l|l|l|l|}
\cline { 2 - 5 } \multicolumn{1}{c|}{} & \multicolumn{2}{c|}{ Live Undiff mESC } & \multicolumn{2}{c|}{ Fixed Undiff mESC } \\
\cline { 2 - 5 } & Hertz model & Capsule model & Hertz model & Capsule model \\
\hline Correlation $r^{2}$ & {$[0.98 \ldots . .0 .99]$} & {$[0.68 \ldots . .0 .90]$} & {$[0.95 \ldots 0.99]$} & {$[0.46 \ldots . .0 .73]$} \\
\hline
\end{tabular}

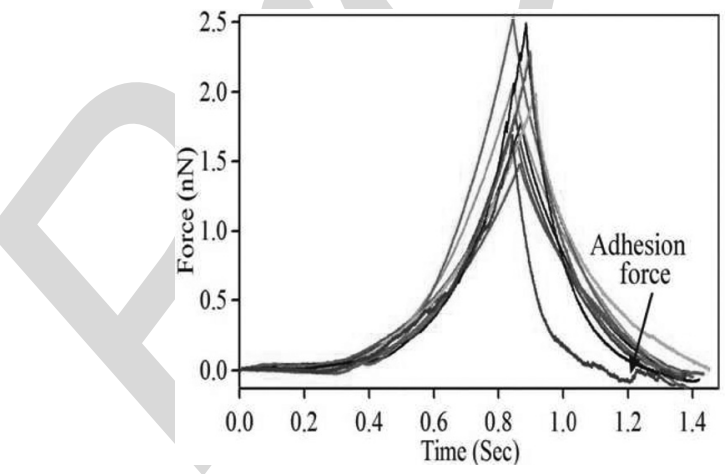

Fig. 16. Load-unload versus time for live undiff.

live and fixed undiff mESC (see Figs. 16- 18). Equation (16) was used to determine the global elastic modulus of the mESC. 470 The average elastic modulus were 17.87 and $0.217 \mathrm{kPa}$ for the 471 fixed and live undiff, respectively, (see Fig. 19). The standard 472 deviations for the fixed and live undiff cells were 3.37 and 473 $0.05 \mathrm{kPa}$. 


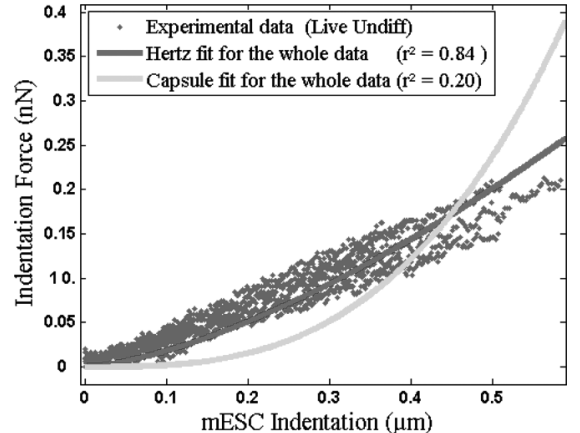

Fig. 17. Live undiff (small deformation).

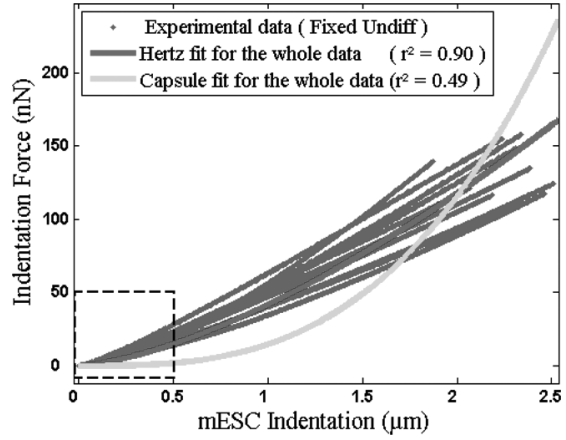

Fig. 18. Fixed undiff mESC.
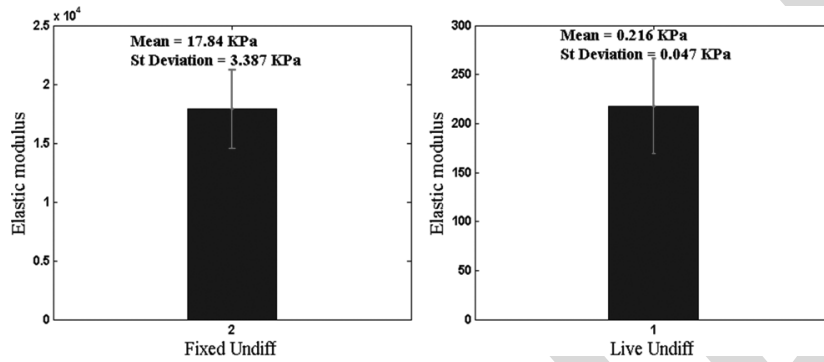

Fig. 19. Elastic modulus for live and fixed undifferentiated mESC calculated from the Hertz contact model for a small deformation (range $0.5 \mu \mathrm{m}$ ) with error bar.
It should be noticed that alterations in the mechanical properties of cells directly reflect changes in their cellular composition, internal structure (cytoskeleton), and external interactions (cellcell and/or cell-surface) that occur during differentiation, aging, and other changes in physiological status that are not accounted in the cell indentation model.

\section{FEM Simulation of CELl INDENTATION}

Fig. 20 presents a photo of the visual and haptic user interface setup. To test the accuracy and reliability of the proposed user interface system with haptics-enabled simulation, a set of experiments is designed using the setup given in Section II. The geometrical dimensions of the cell are determined through AFM image processing and force curve deflection (diameter and height), and the mechanical properties through AFM indentation studies due to the use of an analytical Hertz contact model.

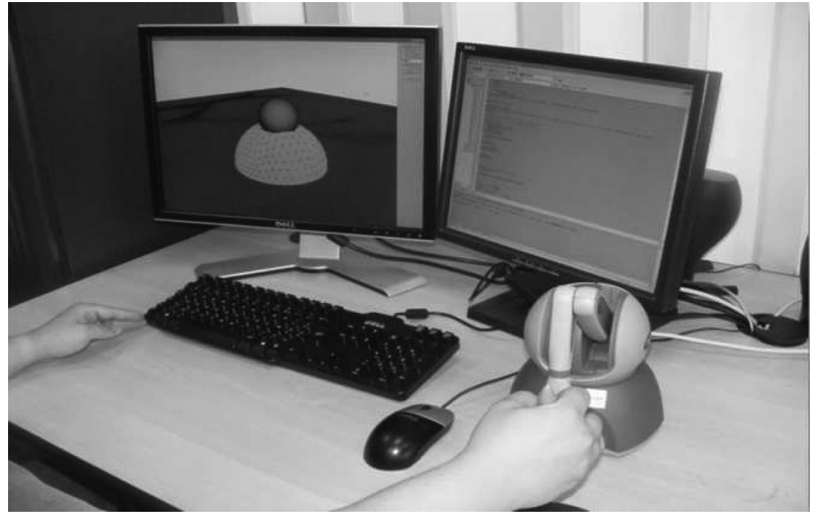

Fig. 20. Real-time indentation simulator using the Omni haptic interface.

TABLE II

Mechanical and Geometrical Properties of Single Cells Selected USED IN THE HAPTICS-ENABLED SIMULATOR

\begin{tabular}{c||cc}
\hline & Live mESC & Fixed mESC \\
\hline \hline Young modulus $(K P a)$ & 0.169 & 26.7 \\
Poisson coefficient & 0.49 & 0.49 \\
Diameter $(\mu \mathrm{m})$ & $\simeq 12$ & $\simeq 12$ \\
Height $(\mu \mathrm{m})$ & 8 & 8 \\
\hline
\end{tabular}

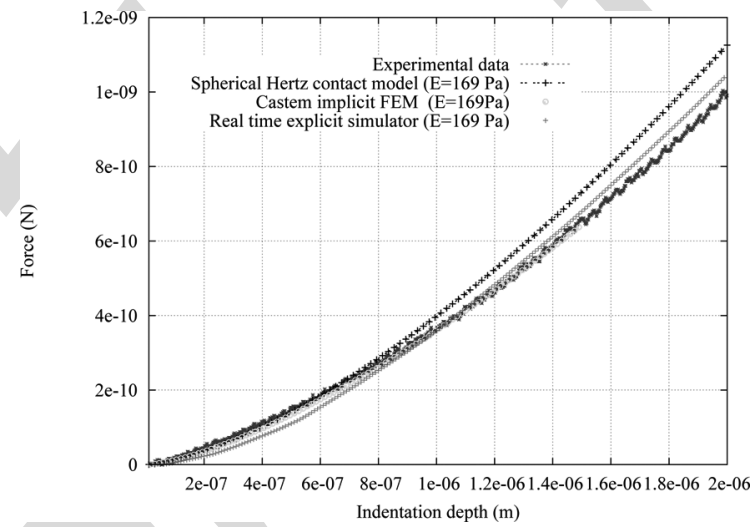

Fig. 21. Force versus indentation for live mESC: comparison between the real-time FEM, the Hertz contact, the quasi-static FEM, and experimental data.

These properties (see Table II) are passed to the finite-element 490 simulations.

In order to demonstrate the validity of our finite-element indentation model in real time, we compare the simulation indentation using the physics-based FEM model to the incremental data provided by AFM indentation tool and to the response simulated with a commercial FE software. Figs. 21 and 22 present the nonlinear relationship between the penetration distance (indentation) and the reaction force for live and fixed undiff cells. The finite-element simulations using the commercial software show good agreement with both Hertz contact model and the experimental indentation data. The developed real-time physicsbased FEM simulator demonstrates its accuracy in predicting 


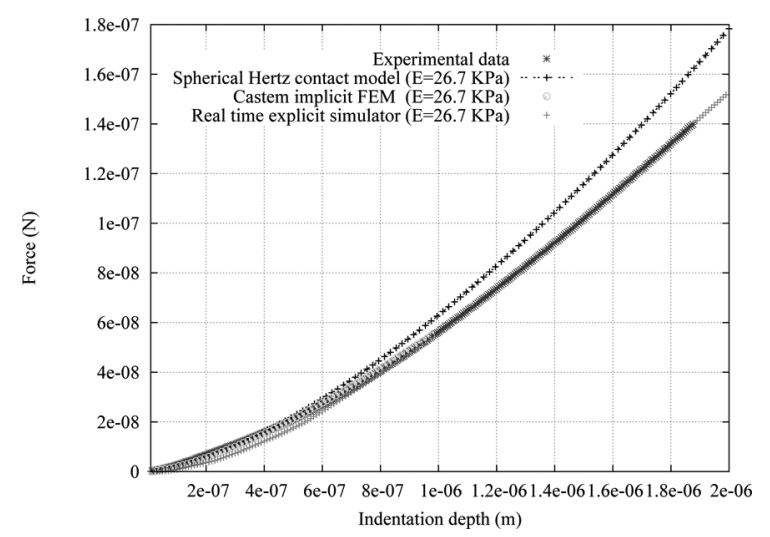

Fig. 22. Force versus indentation for fixed mESC: comparison between the real-time FEM, the Hertz contact, the quasi-static FEM, and experimental data.
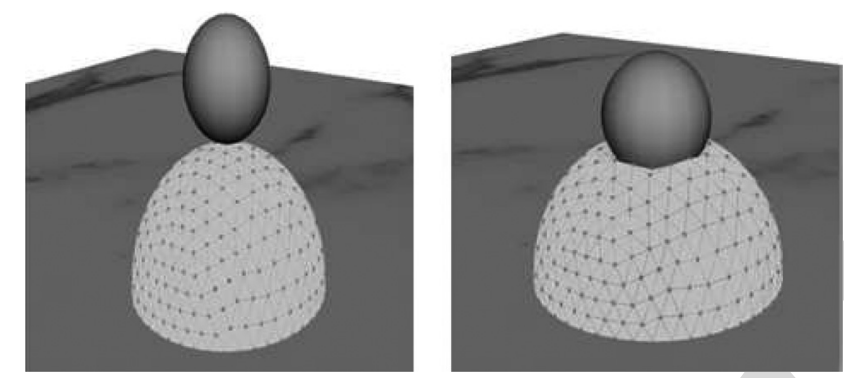

Fig. 23. Simulation of the AFM nanoindentation in VEs. The AFM tip and the stem cell was modeled using finite-element methods. A scene is shown from current training method: the 3-D cell deformation simulation before and after force indentation.

indentation forces for large deformations $(>25 \%)$ of relative nominal strain (see Figs. 21 and 22). Fig. 23 illustrates a realtime cell indentation procedure in a VE for training. It shows a screenshot of the cell surface deformation. The interaction between tip and surface can be visualized to the user in 3-D (sphere and cone represent AFM tip and forces acting on tip, respectively). It shows the indentation (elastic deformation) on the cell surface and repulsive contact forces while pressing on the surface. In order to improve the force feedback rendering, we added a visual metaphor on the computer screen.

The rendered reflection force is depicted by a cone representing the force vector acting on the AFM tip. The visual metaphor has efficiently proved its effectiveness in AFM-based nanomanipulation simulation [23]. The vector amplitude reflects the force feedback amplitude and the vector orientation represents the vertical/horizontal force components. Fig. 24 shows in 3-D real time the probe tip, zoomed cell indentation, and microforces. The indentation force rendered to the operator is composed of a vertical $F_{z}$ and horizontal $F_{x}$ component. For the specific case corresponding to the center indentation, the tangential component is very small $F_{x} \simeq 0$. Only vertical reflection force is provided to the user. He cannot haptically feel it during simulated indentation tests (see Figs. 25(a) and 26). In the case, where the AFM probe is pushed away from the center of the cell, a tangential component exists $\left(F_{x} \neq 0\right)$ and the reflec-

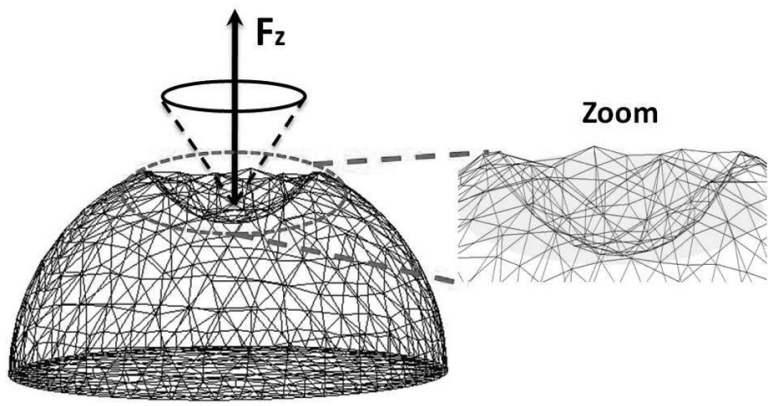

Fig. 24. Depth deformation of the cell: the left picture presents the depth indentation $(2 \mu \mathrm{m})$ of the cell and the right picture shows the zoom of the cell deformation.

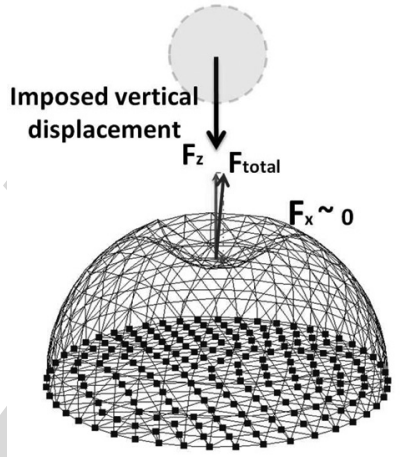

(a)

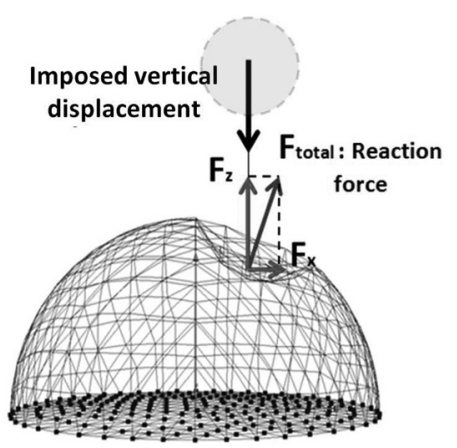

(b)
Fig. 25. Force indentation, where the probe is put (a) in the center and (b) off center of the cell.

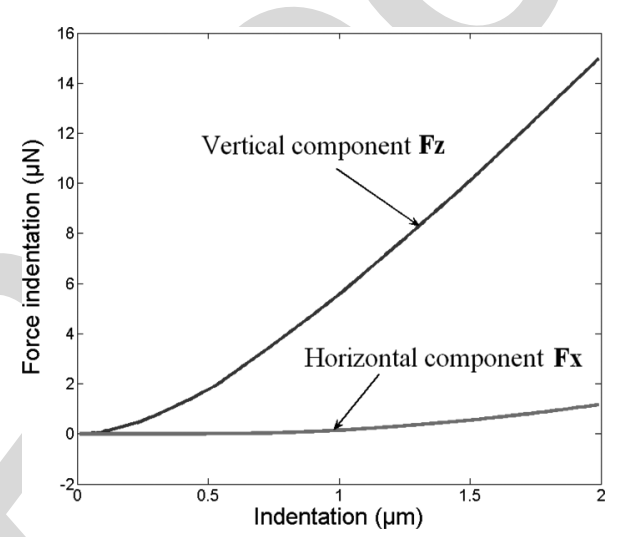

Fig. 26. Horizontal and vertical component force versus indentation: the probe is put in center of the cell.

tion force rendered to the user contains a vertical and tangential $\quad 528$ component (see Figs. 25(b) and 27). We did not reproduce this 529 specific case in our results since the main interest of the paper is 530 to prove experimentally the real-time explicit simulator results 531 during centered cell indentations (since only vertical compo- 532 nents of the force experiments are measured through the AFM 533 cantilever). 


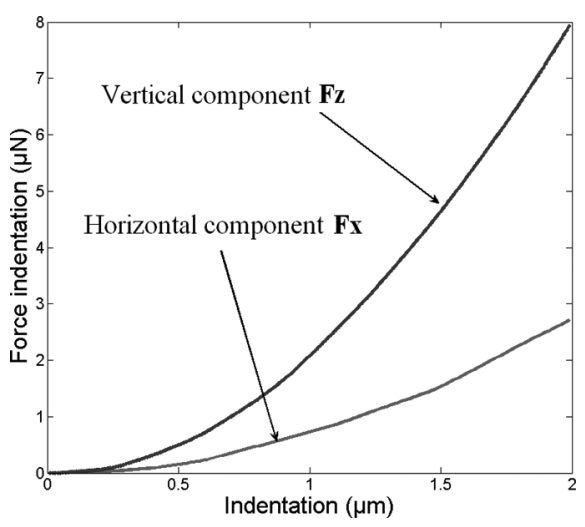

Fig. 27. Horizontal and vertical component force versus indentation: the probe is put off center of the cell.

\section{CONCLUSION}

We have developed a computer-based training system to simulate real-time cell indentation procedures in VEs for training biologists. The simulator provides the user with visual and haptic feedback. The simulation of this procedure involves real-time rendering of computer generated graphical images of the AFM tip, physics-based modeling of soft biological tissue, and display of touch and force sensations to the trainee through the simulation of haptic interactions. The real-time haptics-enabled simulator is realistic, since it is mainly based on experimental AFM data (mechanical and geometrical property of mESC) to accurately replicate the indentation task and predict the cell deformation during indentation. We first investigated the challenging issues in the real-time modeling of the biomechanical properties of the cell indentation through FEMs. Compared to experimental AFM indentation results performed on $\mathrm{mESC}$, we can see clearly that the proposed physically based FEM model is able to simulate the cell deformation through real-time simulation constraints. Currently, we are working on integrating nanoscale effects, such as friction, viscosity, punction, and adhesion forces in the nonlinear FEM model. All modalities will be merged in an ergonomic tool and intelligent biological simulator for stem cell characterization.

\section{REFERENCES}

[1] J. Settleman, "Tension precedes commitment-even for a stem cell," Mol. Cell, vol. 14, pp. 148-150, 2004.

[2] V. E. Meyers, M. Zayzafoon, J. T. Douglas, and J. M. McDonald, "RhoA and cytoskeletal disruption mediate reduced osteoblastogenesis and enhanced adipogenesis of human mesenchymal stem cells in modeled microgravity," J. Bone Miner. Res., vol. 20, pp. 1858-1866, 2005.

[3] R. McBeath, D. M. Pirone, C. M Nelson, K. Bhadriraju, and C. S. Chen, "Cell shape, cytoskeletal tension, and RhoA regulate stem cell lineage commitment," Dev. Cell, vol. 6, pp. 483-495, 2004.

[4] J. Desai, A. Pillarisetti, and A. D. Brooks, "Engineering approaches to biomanipulation," Annu. Rev. Biomed. Eng., vol. 9, pp. 35-53, 2007.

[5] F. Arai, D. Ando, T. Fukuda, Y. Nonoda, and T. Oota, "Micromanipulation based on micro physics, strategy based on attractive force reduction and stress measurement," in Proc. IEEE Int. Conf. Robot. Autom., 1995, pp. 236-241.

[6] A. R. Bausch, W. Moller, and E. Sackmann, "Measurement of local viscoelasticity and forces in living cells by magnetic tweezers," Biophys. J., vol. 76, pp. 573-579, 1999.

[7] T. Ohasti, M. Hagiwara, D. L. Bader, and N. M. Knight, "Intracellular mechanics and mechanotransduction associated with chondrocyte defor- mation during pipette aspiration," Biorheology, vol. 43, no. 3-4, pp. 201214, 2006.

[8] J. L. Alonso and W. H. Goldmann, "Feeling the forces: atmoic force microscopy in cell biology," Life Sci., vol. 72, pp. 2553-2560, 2003.

[9] A. Touhami, B. Nysten, and Y. F. Dufrene, "Nanoscale mapping of the elasticity of microbial cells by atomic force microscopy," Langmuir, vol. 19, pp. 4539-4543, 2003.

[10] E. M. Darling, S. Zauscher, and F. Guilak, "Viscoelastic properties of zonal articular chondrocytes measured by atomic force microscopy," Osteoarthritis Cartilage, vol. 14, pp. 571-579, 2006.

[11] R. E. Mahaffy, S. Park, E. Gerde, J. Kas, and C. K. Shih, "Quantitative analysis of the viscoelastic properties of thin regions of fibroblasts using atomic force microscopy," Biophys. J., vol. 86, pp. 1777-1793, 2004.

[12] J. Alcaraz, L. Buscemi, M. Grabulosa, X. Trepat, B. Fabry, R. Farre, and D. Navajas, "Microrheology of human lung epithelial cells measured by atomic force microscopy," Biophys. J., vol. 84, pp. 2071-2079, 2004.

[13] A. Pillarisetti, C. Keefer, and J. P. Desai, "Mechanical Response of embryonic stem cells using haptics-enabled atomic force microscopy," in Proc. Int. Symp. Experimental Robot., Athens, Greece, 2008.

[14] R. E. Rudd, M. McElfresh, E. Baesu, R. Balhorn, M. Allen, and J. Belak, "Modeling of the Mechanical Deformation of Living Cells in Atomic force Microscopy," in Proc. Int. Conf. Comput. Nanosci., San Francisco, CA, 2003, pp. 23-27.

[15] V. Lulevich, T. Zink, H.-Y. Chen, F.-T. Liu, and G.-Y. Liu, "Cell mechanics using atomic force microscopy-based single-cell compression," Langmuir, vol. 22, no. 19, pp. 8151-8155, 2006.

[16] M. Bro-Nielsen and S. Cotin, "Real-time volumetric deformable models for surgery simulation using finite elements and condensation," Comput. Graph. Forum (Eurographics), vol. 5, no. 3, pp. 57-66, 1996.

[17] S. Cotin, H. Delingette, and N. Ayache, "A hybrid elastic model for realrime cutting, deformations, and force feedback for surgery training and simulation," Vis. Comput., vol. 16, pp. 437-452, 2000.

[18] J.-M. Schwartz, M. Dellinger, D. Rancourt, C. Moisan, and D. Laurendeau, "Modeling liver tissue properties using a non-linear visco-elastic model for surgery simulation," Med. Image Anal., vol. 9, no. 2, pp. 103-112, 2005.

[19] H. Ladjal, J.-L. Hanus, A. Pillarisetti, C. Keefer, A. Ferreira, and J. P Desai, "Atomic force microscopy-based single-cell indentation: Experimentation and finite element simulation," in Proc. IEEE Int. Conf. Robots Intell. Syst., St. Louis, MO, Oct. 11-15 2009.

[20] A. Pillarisetti, H. Ladjal, C. Keefer, A. Ferreira, and J. P. Desai, "Mechanical characterization of mouse embryonic stem cells," in Proc. 31st Ann Int. IEEE EMBS Conf., Minnesota, MN, Sep. 2-6 2009.

[21] A. Pillarisetti, C. Keefer, and J. P. Desai, "Mechanical response of embryonic stem cells using haptics-enabled atomic force microscopy," in Proc. Int. Symp. Exp. Robot., Athens, Greece, 2008.

[22] V. V. Lulevich, D. Andrienko, and O. I. Vinogradova, "Elasticity of polyelectrolyte multilayer microcapsules," J. Chem. Phys., vol. 120, no. 8, pp. 3822-3826, 2004.

[23] W. Vogl, K.-L.-Ma. Bernice, and M. Sitti, "Augmented reality user interface for an atomic force microscope-based nanorobotic system," IEEE Trans. Nanotechnol., vol. 5, no. 4, pp. 397-406, Jul. 2006.

[24] R. Adams, M. Moreyra, and B. Hannaford, "Stability and performance of haptic displays: Theory and experiments," in Proc. ASME Winter Annu. Meet. Haptics Workshop, Anaheim, CA, 1998.

[25] F. B. Llewellyn, "Some fundamental properties of transmission systems," Proc. IRE, vol. 40, 1952.

[26] D. Maugis, Contact, Adhesion and Rupture of Elastic Solids, Springer 2000.

[27] H. Ladjal, J.-L. Hanus, A. Pillarisetti, C. Keefer, A. Ferreira, and J. P. Desai, "Realistic visual and haptic feedback simulator for real-time cell indentation," in Proc. IEEE Int. Conf. Robots Intell. Syst., Taipei, Taiwan, Oct. 18-22 2010.

[28] Y. Cao, D. Yang, and W. Soboyejoy, "Nanoindentation method for determining the initial contact and adhesion characteristics of soft polydimethylsiloxane," J. Mat. Res., vol. 20, no. 8, pp. 2004-2011, 2005.

[29] M. Girot, M. Boukallel, and S. Régnier, "Modeling soft contact mechanism of biological cells using an atomic bio-microscope," in Proc. Int Conf. Intell. Robots Syst., Beijing, China, 2006, pp. 1831-1836.

[30] E. U. Azeloglu and K. D. Costa, "Dynamic AFM elastography reveals phase dependent mechanical heterogeneity of beating cardiac myocyte," in Proc. IEEE Conf. Eng. Med. Biol. Soc., 2009, pp. 7180-7183.

[31] C. L. Kao, L. K. Tai, S. H. Chiou, Y. J. Chen, K. H. Lee, S. J. Chou, Y. L. Chang, C. M. Chang, S. J. Chen, H. H. Ku, and H. Y. Li, "Resveratrol 
promotes osteogenic differentiation and protects against dexamethasone damage in murine induced pluripotent stem cells," Stem Cells Dev., vol. 19, no. 2, pp. 247-258, Feb. 2010.

[32] H. Xie and S. Régnier, "Development of a flexible robotic system for multiscale applications of micro/nanoscale manipulation and assembly," IEEE/ASME Trans. Mechatronics, 2010.

[33] R. Perez, J. Agnus, C. Clevy, A. Hubert, and N. Chaillet, "Modeling, fabrication, and validation of a high-performance 2-DoF piezoactuator for micromanipulation," IEEE/ASME Trans. Mechatronics, vol. 10, no. 2, pp. 161-171, Apr. 2005.

[34] C. Elbuken, M. B. Khamesee, and M. Yavuz, "Design and implementation of a micromanipulation system using a magnetically levitated MEMS robot," IEEE/ASME Trans. Mechatronics, vol. 14, no. 4, pp. 434-445, Aug. 2009.

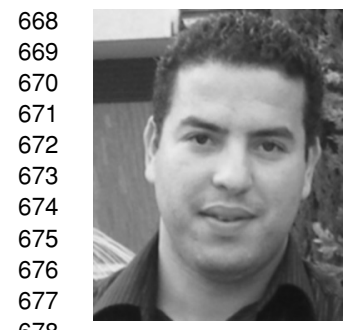

Hamid Ladjal received the Engineer degree in electronics and computing from Houari Boumediène University of Science and Technology, Algeria, in 2000, the Master's degree in virtual reality and complex systems from Evry-Val d'Essone University, Evry, France, and the Ph.D. degree in robotics from the University of Orléans, Orléans, France, in 2010.

$\mathrm{He}$ is currently an Associate Professor in the Institut PRISME, Ecole Nationale Supérieure d'Ingénieurs de Bourges, Bourges, France. His research interests include modeling and finite-element 679 simulation, visual and haptic feedback, reality-based soft-tissue modeling for 680 real-time simulation, image processing, control and micromanipulation, biological cell characterization, and cell biomechanics.

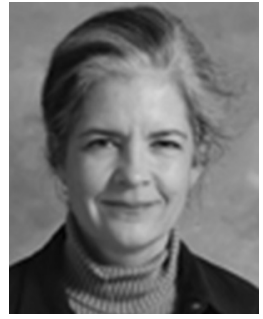

Carol Keefer is currently an Associate Professor in the Department of Animal and Avian Sciences, University of Maryland, College Park, MD, where she has established the new Biotechnology Program in Animal Sciences. Her laboratory explores mechanisms controlling development in mammalian embryos and embryonic stem cells and hopes to apply knowledge gained to the development of biomedical models of human and animal disease and to the production of therapeutic proteins via transgenic animals (biopharming). She was also a Senior Research Scientist at Nexia Biotechnologies Inc., Montreal, QC, Canada.

Prof. Keefer is a member of the Editorial Board of Cellular Reprogramming (formerly Cloning and Stem Cells). She is an active member of the Society for the Study of Reproduction (SSR). She was President of the International Embryo Transfer Society (IETS).

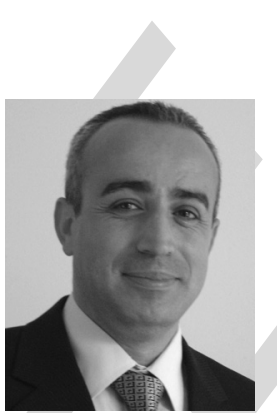

Antoine Ferreira (M'04) received the M.S. and $\mathrm{Ph} . \mathrm{D}$. degrees in electrical and electronics engineering from the University of Franche-Comté, Besancon, France, in 1993 and 1996, respectively.

In 1997, he was a Visiting Researcher in the ElectroTechnical Laboratory, Tsukuba, Japan. He is currently a Professor of robotics engineering at the Institut PRISME, Ecole Nationale Supérieure d'Ingénieurs de Bourges, Bourges, France. He is an author of three books on micro- and nanorobotics and more than 100 journal and conference papers

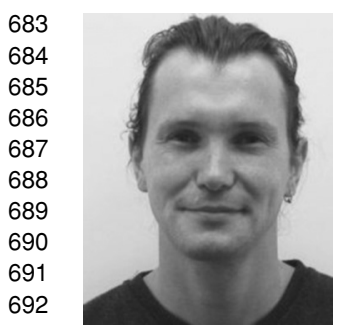

Jean-Luc Hanus received the M.S. degree from the National Aerospace and Mechanical Enginneering School, France, in 1993, and the Ph.D. degree in mechanical engineering from the University of Poitiers, Poitiers, France, in 1999.

$\mathrm{He}$ is currently an Assistant Professor of mechanical engineering at the Bourges National Engineering School, Bourges, France. His research interests include the area of nonlinear mechanical models and efficient numerical methods in dynamic analysis of biological cell responses.

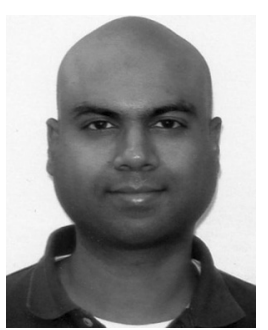

Anand Pillarisetti received the Undergraduate degree from the National Institute of Technology, Warangal, India, in 2002, the M.S. degree in mechanical engineering and mechanics from Drexel University, Philadelphia, PA, in 2006, and the Ph.D. degree in mechanical engineering from University of Maryland, College Park, MD, in 2008.

$\mathrm{He}$ is currently a Postdoctoral Researcher in the Department of Mechanical Engineering and Applied Mechanics, University of Pennsylvania, Philadelphia. His research interests include sensors, microelectromechanical systems, atomic force microscopy, and nanotechnology. and book contributions. His research interests include the design, modeling, and control of micro and nanorobotic systems using active materials, micronanomanipulation systems, biological nanosystems, and bionanorobotics.

Dr. Ferreira was the Guest Editor for different special issues of the IEEE/ASME TRANSACTIONS ON MECHATRONICS in 2009, International Journal of Robotics Research in 2009, and the IEEE Nanotechnology Magazine in 2008.

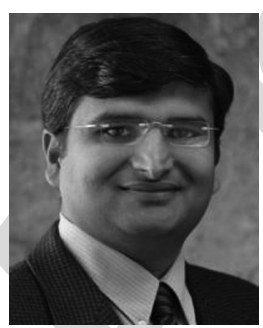

Jaydev P. Desai (SM'07) received the B.Tech. degree 744 from the Indian Institute of Technology, Bombay, 745 India, in 1993, and the M.A. degree in mathemat- 746 ics in 1997, and the M.S. and Ph.D. degrees in me- 747 chanical engineering and applied mechanics, in 1995748 and 1998, respectively, all from the University of 749 Pennsylvania, Philadelphia, PA.

$\mathrm{He}$ is currently an Associate Professor in the De- 751 partment of Mechanical Engineering, University of 752 Maryland, College Park, MD, where he is also the Di- 753 rector of the Robotics, Automation, and Medical Sys- 754 tems Laboratory. His research interests include image-guided surgical robotics, 755 haptics, reality-based soft-tissue modeling for surgical simulation, model-based 756 teleoperation in robot-assisted surgery, and cellular manipulation.

Prof. Desai was the recipient of an NSF CAREER Award, a Lead Inventor 758 on the "Outstanding Invention of 2007 in Physical Science Category" at the 759 University of Maryland, and the Ralph R. Teetor Educational Award. He is 760 currently a member of the Haptics Symposium Committee, the Co-Chair of 761 the Surgical Robotics Technical Committee of the IEEE Robotics and Automa- 762 tion Society, and a member of the Editorial Board of the IEEE TRANSACTIONS 763 ON BIOMEDICAL ENGINEERING, ASME Journal of Medical Devices, and IEEE 764 TRANSACTIONS ON INFORMATION TECHNOLOGY IN BIOMEDICINE. He is also a 765 member of the American Society of Mechanical Engineers. 政 740 741 708

\section{1} 12 15 17 (a) 720 721 722 26 28 729 730 731

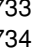
, 38
39 42 is 
Q1: Author: Please check and confirm footnote first as typeset.

Q2. Author: Please check and confirm the citation for Figs. 9 and 10 have been interchanged, with their respective captions, in 770 order to maintain sequential order in the text.

Q3. Author: Please spell out "JKR" and "DMT" in full, if possible.

Q4. Author: Please check and confirm in this sentence "Figs. 14 and 15 show......indentation range." the citations for Figs. 14 773 and 15 have been interchanged.

Q5. Author: Please check the citations for Figs. 16- 18 as typeset.

Q6. Author: Please provide page range for ref. [13], [19]-[21], [27].

Q7. Author: Please provide page range for Ref. [25].

Q8. Author: Please update Ref. [32].

Q9. Author: Please provide the title of Engineer and Master's degrees for H. Ladjal.

Q10. Author: Please provide the title of Undergraduate degree for A. Pillarisetti.

Q11. Author: Please provide the title (B.Sc., M.Sc., Ph.D., etc.) and university names from which C. Keefer received these degrees. 781 